\title{
Programmatic and Constructive Interdependence: Emerging Insights and Predictive Indicators of Development Resource Demand
}

\author{
Robert M. Flowe \\ Mark Kasunic \\ Mary Maureen Brown \\ Paul L. Hardin III \\ James McCurley \\ David Zubrow \\ William Anderson
}

July 2010

TECHNICAL REPORT

CMU/SEI-2010-TR-024

ESC-TR-2010-024

Software Engineering Measurement and Analysis

Unlimited distribution subject to the copyright.

http://www.sei.cmu.edu

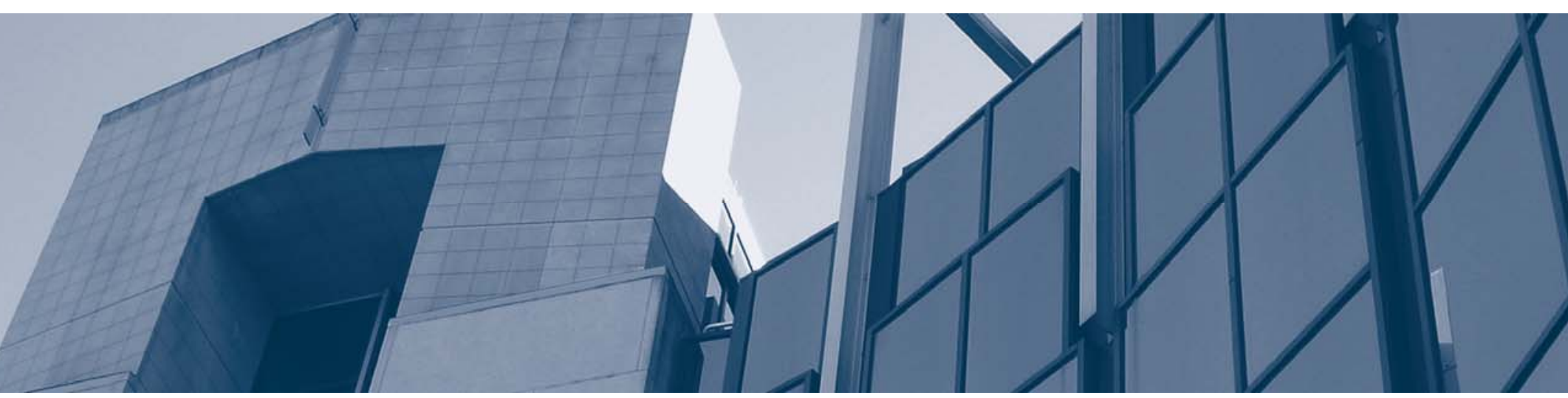


This report was prepared for the

SEI Administrative Agent

ESC/XPK

5 Eglin Street

Hanscom AFB, MA 01731-2100

The ideas and findings in this report should not be construed as an official DoD position. It is published in the interest of scientific and technical information exchange.

This work is sponsored by the U.S. Department of Defense. The Software Engineering Institute is a federally funded research and development center sponsored by the U.S. Department of Defense.

Copyright 2010 Carnegie Mellon University.

\section{NO WARRANTY}

THIS CARNEGIE MELLON UNIVERSITY AND SOFTWARE ENGINEERING INSTITUTE MATERIAL IS FURNISHED ON AN “AS-IS” BASIS. CARNEGIE MELLON UNIVERSITY MAKES NO WARRANTIES OF ANY KIND, EITHER EXPRESSED OR IMPLIED, AS TO ANY MATTER INCLUDING, BUT NOT LIMITED TO, WARRANTY OF FITNESS FOR PURPOSE OR MERCHANTABILITY, EXCLUSIVITY, OR RESULTS OBTAINED FROM USE OF THE MATERIAL. CARNEGIE MELLON UNIVERSITY DOES NOT MAKE ANY WARRANTY OF ANY KIND WITH RESPECT TO FREEDOM FROM PATENT, TRADEMARK, OR COPYRIGHT INFRINGEMENT.

Use of any trademarks in this report is not intended in any way to infringe on the rights of the trademark holder.

Internal use. Permission to reproduce this document and to prepare derivative works from this document for internal use is granted, provided the copyright and "No Warranty" statements are included with all reproductions and derivative works.

External use. This document may be reproduced in its entirety, without modification, and freely distributed in written or electronic form without requesting formal permission. Permission is required for any other external and/or commercial use. Requests for permission should be directed to the Software Engineering Institute at permission@sei.cmu.edu.

This work was created in the performance of Federal Government Contract Number FA8721-05-C-0003 with Carnegie Mellon University for the operation of the Software Engineering Institute, a federally funded research and development center. The Government of the United States has a royalty-free government-purpose license to use, duplicate, or disclose the work, in whole or in part and in any manner, and to have or permit others to do so, for government purposes pursuant to the copyright license under the clause at 252.227-7013.

For information about SEI publications, please visit the library on the SEI website (www.sei.cmu.edu/library). 


\section{Table of Contents}

Executive Summary

Abstract

ix

1 Introduction

1.1 Background

1.2 The Problem

1.3 Our Hypothesis

1.4 Research Approach

2 Study 1: Identification of Diagnostic Risk Indicators Associated with Integration and Interoperability

2.1 Research Question

2.2 Background 5

2.3 Method 5

2.4 Results and Discussion 5

$3 \quad$ Study 2: Using DAES-SARS Information for Forecasting Program Performance 7

$\begin{array}{ll}3.1 & \text { Research Question }\end{array}$

3.2 Background 7

3.3 Method 8

3.4 Results and Discussion 8

$4 \quad$ Study 3: Exploring the Extent of Programmatic Interdependence 15

4.1 Research Question $\quad 15$

4.2 Background 15

4.3 Method 15

$\begin{array}{ll}4.4 & \text { Results and Discussion } \\ \end{array}$

$5 \quad$ Study 4: Using Network Methods to Explore the Evolution of Acquisition Program Interdependence Over Time $\quad 19$

5.1 Research Question 19

5.2 Background 19

5.3 Method 19

$\begin{array}{lll}5.4 & \text { Results and Discussion } & 20\end{array}$

6 Study 5: Using Network Methods to Explore the Relationship Between Systems Development Interdependencies and Development Resources 25

$\begin{array}{ll}6.1 & \text { Research Question } \\ 6 & 25\end{array}$

$\begin{array}{ll}6.2 \text { Background } & 25\end{array}$

6.3 Method 26

6.3.1 Measuring Constructive Interdependence 26

6.3.2 Measuring Development Resource Demand 27

$\begin{array}{ll}\text { 6.4 Results and Discussion } & 27\end{array}$

6.4.1 Part I - Relationship between Total Number of System Nodes and
Integration Density

6.4.2 Part II - Relationship Between Constructive Interdependence and
Development Resource Demand

6.4.3 Part III - Development of the Equivalent Node Measure 30

7 Summary $\quad 35$

i | CMU/SEI-2010-TR-024 
Appendix A: Diagnostic Risk Indicators Associated with Integration and Interoperability

Appendix B: Additional Analyses Using DAES-SARS Information for Forecasting Performance

References/Bibliography 


\section{List of Figures}

Figure 1: $\quad$ Cause and Effect Relationships 2

Figure 2: Interdependencies at One Dimension Drive Interdependencies at Another 3

Figure 3: Mapping of Interdependencies Among all DAES Programs 16

Figure 4: Mapping of Interdependencies Among ACAT I Programs 16

Figure 5: Number of MDAPs per Program Element from 1997 to 2007

Figure 6: Program Clusters as of 1997

Figure 7: Program Clusters as of 2007

Figure 8: Example - Calculating Average Degree 23

Figure 9: Average Program Network Degree by Year 23

Figure 10: Example: Node and Link Counting Rules Applied to ISP Documents 27

Figure 11: Integration Density of MDAPs 28

Figure 12: Development Resource Demand Versus Number of Nodes 29

Figure 13: Development Resource Demand Versus Number of Links 29

Figure 14: Fitted Equation of Equivalent Nodes to Development Resource Demand 32

Figure 15: Sample Earned Value Management Report 43 
iv | CMU/SEI-2010-TR-024 


\section{List of Tables}

Table 1: $\quad$ The Three Dimensions of Interdependence 3

Table 2: $\quad$ DAES, DAES Assessment Indicators, and SAR Breach Data 9

Table 3: $\quad$ Combined Indicators 10

$\begin{array}{ll}\text { Table 4: } \quad \text { Isolated Indicators } & 11\end{array}$

Table 5: $\quad$ Schedule Breaches Predicted by Schedule Indicators 12

Table 6: $\quad$ Program Elements and MDAP Programs 1997-2007 20

Table 7: $\quad$ Description of Network Terms Used for Analysis 26 
vi | CMU/SEI-2010-TR-024 


\section{Executive Summary}

This report describes a series of ongoing research efforts investigating the role of interdependence in the acquisition of Major Defense Acquisition Programs (MDAPs). This research initiative was sponsored by the Office of the Secretary of Defense (OSD).

The overall goal of the research was to identify, quantify, and assess the degree of programmatic and constructive interdependence and to assess the effects of interdependence on program risk.

This paper reports the results of five research studies that were conducted from 2004 to 2009.

Study 1 explored the qualitative factors that confound program cost and schedule estimation.

Study 2 employed data-mining and statistical analyses to determine whether Defense Acquisition Executive Summary (DAES) reports and Select Acquisition Reports (SARs) can be used to forecast program performance. An interesting result from this study is that there was no evidence that such indicators are effective in predicting program breaches.

Studies 3-5 employed network analysis techniques to quantitatively characterize programmatic and constructive interdependencies in the acquisition enterprise. These last three studies culminated in graphical models that relate interdependence and program cost.

Four critical findings were revealed by this research.

1. Our research study found no evidence that indicators reported within DAES reports or SARs predict program breach events.

2. Limiting the definition of interdependence to programs that are identified and funded as joint programs is insufficient. All programs are interdependent to some degree, and therefore interdependence-related risk is widespread, regardless of service affiliation or participation.

3. Continued investment in the gathering of objective, authoritative data should be a major emphasis across the Department of Defense (DoD). The consequence of failing to gather and analyze these important data is substantial program failure, wasted resources, and erosion of the public trust in the integrity and capability of the DoD.

4. The traditional methods of analyzing risk, while important, need to be supplemented with network analysis techniques to reveal the true scope and effects of programmatic and constructive interdependence. Additional investigation into the methods and measures that can reveal critical interdependencies is clearly warranted. 
viii | CMU/SEI-2010-TR-024 


\section{Abstract}

The challenges program managers encounter in attempting to deliver programs on time and on budget are well substantiated. A significant driver of the turbulence experienced by acquisition programs today is the transformation to joint capabilities. This report describes a series of ongoing research efforts, sponsored by the Office of the Secretary of Defense (OSD), that investigated the role of interdependence in the acquisition of major defense acquisition programs.

The overall goal of the research was to identify, quantify, and assess the degree of programmatic and constructive interdependence and to assess the effects of interdependence on program risk. A number of important findings and noteworthy insights were discovered as programs were examined in light of their interdependencies with other programs. The results indicate that an expanded definition of interdependencies along with the incorporation of network analysis tools may provide important insights into program performance in a joint capability arena. 
x | CMU/SEI-2010-TR-024 


\section{Introduction}

\subsection{Background}

The challenges program managers encounter in attempting to deliver programs on time and on budget are well substantiated. A significant driver of the turbulence experienced by acquisition programs today is the transformation to joint capabilities. With the end of the Cold War, and the erosion of the relatively stable Eastversus-West power structure, multi-lateral, state- and non-state-affiliated threats emerged. Strategic and operational advantage shifted from mass and firepower to agility and precision [CJCS 2000].

This need for integrated joint operations triggered corresponding changes in acquisition policy and organizational processes. A variety of initiatives have been enacted that are intended to rectify the situation. These include the Grace Commission [Grace 1984], the Packard Commission [Packard 1986], the GoldwaterNichols Act of 1986 [Lederman 1999], the Defense Acquisition Performance Assessment [Kadish 2006], and multiple Defense Science Boards and GAO reports. In addition, pending legislation intends to impose further restructuring of defense acquisition processes, organizations, and the workforce [Doyle 2009].

Secretary of Defense Donald Rumsfeld's call for transformation drove new organizational performance goals that stressed adaptive planning, accelerated acquisition cycles, output-based management, and a reformed analytic support agenda. Continuing on this theme, Secretary of Defense Robert Gates has encouraged “jointness." Documents such as the Capstone Concept for Joint Operations [CJCS 2009] aim to both inform and equip military leaders with the ability to meet future threats. In recognition that integration doesn't "just happen," the DoD created linkages between joint operational capabilities and the DoD acquisition system such as those described through the Joint Capabilities Integration and Development System (JCIDS) [CJCSI 2005] and the various DoD interoperability standards [CJCSI 2008, DoDD 2004].

However, despite vigorous acquisition reform, oversight, and scrutiny, cost overruns and schedule delays remain unacceptably high. Fundamentally similar issues continue to plague the DoD acquisition process. This leads us to ask whether we have identified the root of the underlying problem that drives the observed behaviors.

\subsection{The Problem}

Adjusting to the needs of joint capabilities has not been an easy task, and organizational theorists do not have much empirical advice to offer [Provan 2007, Olsen 2005, Meier 2008, Agranoff 2003].

The fact remains that the transformational mandate of top DoD leadership to achieve joint capabilities is superimposed on a foundation reflecting the traditional service-led, program-centric acquisition paradigm. Experience over the past decade demonstrates that the institutional focus on the program as the principal management mechanism tends to blunt awareness of inter-programmatic issues, such as integration and interoperability, particularly when these issues cross service or other organizational boundaries.

The effects of this discontinuity have not been fully articulated, but it is striking that in an era where jointness, interoperability, and capabilities-based focus are dominant themes, the acquisition process is still measured and evaluated primarily from a program-centric perspective [GAO 2009]. Therefore, it is perhaps not surprising that the response to the perceived shortfalls in the acquisition process is addressed in a largely program-centric way. 
For the acquisition community and its stakeholders, a fundamental paradigm shift would be the recognition that individual programs are not isolated and sovereign but are inextricably interdependent with other programs and multiple external factors that resist program-specific, control-based strategies. For improvement efforts to be effective where prior efforts have failed, they must develop different perspectives to augment the prevailing program-centric paradigm and reveal the "hidden" drivers of program behaviors. By and large, the study of interdependence and its effects on government programs remains in its infancy. Clearly, further applied research in systems development and acquisition management of integrated capabilities is necessary.

\subsection{Our Hypothesis}

The central hypothesis that steered our research efforts is that interdependence is a significant contributor to the behavior and performance of acquisition programs.

As illustrated in Figure 1, operational demand for joint capabilities establishes the need for interoperability. Interoperability, by definition, entails interactions among two or more entities — and in the implementation of these interactions, interdependencies are necessarily created. These resultant interdependencies must be addressed and serviced through integration activities. It is the demands of these interdependencies that impinge on programs by driving the expenditure of effort (integration activities) and the incurrence of cost and schedule overruns.

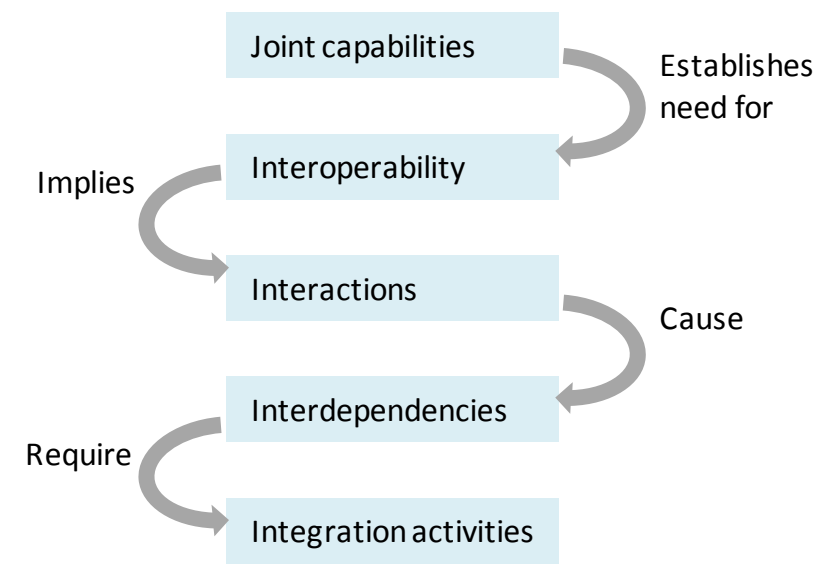

Figure 1: Cause and Effect Relationships

We propose that interdependence can be examined in light of three distinct domains: programmatic, constructive, and operational. ${ }^{1}$ The three dimensions of interdependence are characterized in Table 1.

1 Use of these constructs (programmatic, constructive, and operational views) was adapted from the System of Systems Interoperability (SOSI) model [Morris 2004]. 
Table 1: The Three Dimensions of Interdependence

\begin{tabular}{ll}
\hline $\begin{array}{l}\text { Domains of } \\
\text { Interdependence }\end{array}$ & Description \\
\hline Programmatic & $\begin{array}{l}\text { Encompasses the activities related to the management of one program } \\
\text { in the context of other programs and includes all acquisition, financial, } \\
\text { and program management activities that govern the lifecycle of the } \\
\text { systems that support end-user needs. }\end{array}$ \\
\hline Constructive & $\begin{array}{l}\text { This dimension is the nuts and bolts of what we commonly think of as } \\
\text { systems and software engineering. It addresses technologies (and the } \\
\text { technical activities to select and apply them). These technologies } \\
\text { commonly include shared architectural elements, data specifications, } \\
\text { communication protocols, and common standards. }\end{array}$ \\
\hline Operational & $\begin{array}{l}\text { Refers to the activities related to the actual operation of a system by the } \\
\text { end user in the context of others systems. }\end{array}$ \\
\hline
\end{tabular}

As illustrated in Figure 2, interdependencies are driven into the supporting programmatic structures of the acquisition domain through corresponding interdependencies among the systems to be developed, in response to operational user needs.

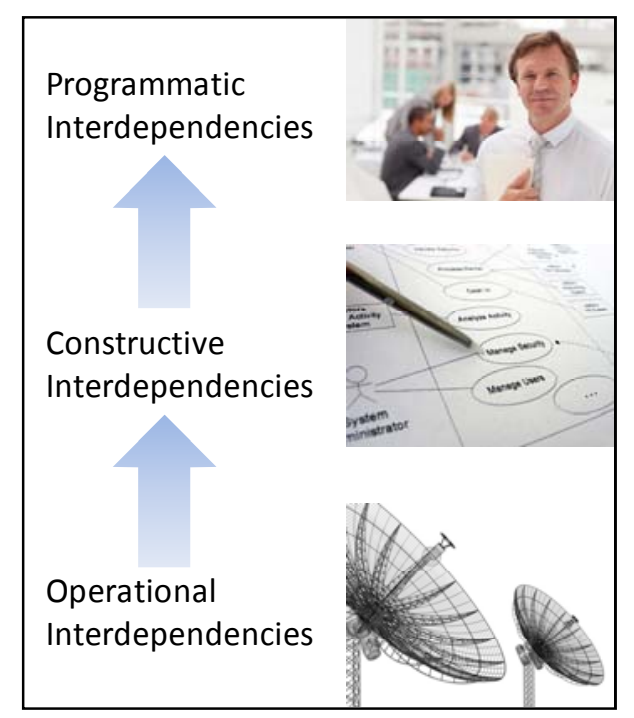

Figure 2: Interdependencies at One Dimension Drive Interdependencies at Another

\subsection{Research Approach}

Having postulated that interdependence may be a significant contributor to cost and schedule estimation error, the following research questions were proposed and investigated:

- What are the qualitative factors that contribute to system cost and schedule estimation error?

- What is the efficacy of using program oversight information to mitigate problems and predict breaches?

- How extensive is programmatic interdependence?

- Is programmatic interdependence increasing over time? 
- What is the relationship between constructive interdependence and a program's development resource demand?

To address these questions, the following five research studies listed below were conducted from 2004 to 2009 .

\begin{tabular}{lll}
\hline Study & Description & See Page \\
\hline 1 & $\begin{array}{l}\text { Identification of Diagnostic Risk Indicators Associated with Integration and } \\
\text { Interoperability }\end{array}$ & 5 \\
\hline 2 & Using DAES-SARS Information for Forecasting Program Performance & 7 \\
\hline 3 & \begin{tabular}{l} 
Exploring the Extent of Programmatic Interdependence \\
\hline 4
\end{tabular} & $\begin{array}{l}\text { Using Network Methods to Explore the Evolution of Acquisition Program } \\
\text { Interdependence Over Time }\end{array}$ \\
\hline 5 & $\begin{array}{l}\text { Using Network Methods to Explore the Relationship between Systems } \\
\text { Development Interdependencies and Development Resources }\end{array}$ & 25 \\
\hline
\end{tabular}

A description of each research study follows. This paper concludes with a summary section followed by appendices that are referred to within some of the study descriptions. 


\section{Study 1: Identification of Diagnostic Risk Indicators Associated with Integration and Interoperability}

\subsection{Research Question}

What are the qualitative factors that contribute to system cost and schedule estimation error?

\subsection{Background}

Having postulated that interdependence might be a significant contributor to the behavior and performance of acquisition programs, we initially examined the qualitative factors that we believe contribute to errors associated with system cost and schedule estimation. This led to identification of a set of categorized diagnostic risk factors that we believe impact system cost and schedule performance. As some portion of program risk often matures into program problems, we assume a corresponding relationship to program cost and schedule estimation error.

\subsection{Method}

The approach was to conduct a literature search, review survey information, and to interview subject matter experts.

\subsection{Results and Discussion}

A list of risk indicators was synthesized from research and then grouped into four categories:

1. Missing Requirements (constructive issues)

2. Organizational \& Institutional Obstacles (programmatic issues)

3. Lifecycle Sustainment (operational issues)

4. Team Performance (the ability of teams to address constructive, programmatic, and operational issues that arise)

The categorized risk indicators are presented in Appendix A.

These results are presented as "rules of thumb" guidance that can alert an analyst or manager about potential program risk.

Each risk statement is accompanied by a plus (+) or minus (-) sign to indicate the types of characteristics that we believe mitigate $(+)$ or exacerbate (-) the associated risk of a particular heuristic. The guidance does not provide a scoring algorithm for the set of heuristics.

We believe this guidance will resonate with experienced software-oriented program management and that these findings are relevant to broader topic areas in systems engineering. 
6 | CMU/SEI-2010-TR-024 


\section{Study 2: Using DAES-SARS Information for Forecasting Program Performance}

\subsection{Research Question}

What is the efficacy of using program oversight information to mitigate problems and predict breaches?

\subsection{Background}

This study examines the efficacy of using the program oversight information reported to the Office of the Secretary of Defense (OSD) acquisition community to mitigate problems and predict breaches. Project managers submit required information to acquisition organizations in the U.S. Department of Defense (DoD), which is then collected in the Defense Acquisition Executive Summary (DAES), published on a quarterly basis, and the Select Acquisition Report (SAR), published on an annual basis. Using DAES reports and SARs, program development issues can be examined on a quarterly basis using standardized variables to indicate risk. DAES reports also form a major component of acquisition oversight, with appropriate defense agencies adding their assessment of the risk indicators to the Defense Acquisition Management Information Retrieval (DAMIR) database.

In this study, we examined a single major defense acquisition program (MDAP) that suffered multiple breach events over a 12-year period. This program-the Multifunctional Information Distribution System-Low Volume Terminal (MIDS-LVT) program - began as a concept in the 1970s, became an international military cause célèbre during the 1980s, went into engineering and manufacturing development during the 1990s, and began full-rate production after 2003. The current program plan continues with production and deployment until 2012. This study uses MIDS-LVT data from 1997-2006.

The MIDS-LVT program was selected as our target program due to its longevity, complexity, the availability of unclassified data, and the many SoS characteristics it had because of its multinational composition. We examined the MIDS-LVT program to see how well information generated for the DAES reports corresponded to cost and schedule breaches during the program life cycle.

The DAES reports and SARs are the major sources of ongoing information regarding program performance reported to Pentagon and acquisition authorities, and their usefulness relies heavily on the program manager's ability to communicate relevant and useful information. DoD 5000.x mandates the use of particular fields for these reports, which leads to consistency of data over time. In an attempt to stay ahead of the game and manage emerging issues that impact cost and schedule-particularly breaches-the Program Assessment Indicators of the DAES reports require explanations by the program manager in the following categories:

- performance characteristics

- test and evaluation

- logistics requirements and readiness objectives

- cost

- funding

- schedule 
- contracts

- $\quad$ production

- $\quad$ management structure

- $\quad$ interoperability

Each indicator is assigned a ranking of green, green advisory, yellow, yellow advisory, or red, depending on the assessed severity of issues in each category. If sufficient information was encapsulated by the G-Y-R evaluations, we hypothesized that there would be a correlation between the assessment scores and the occurrence of breaches.

\subsection{Method}

Information was extracted from all available MIDS-LVT DAES reports regarding the program assessment indicators. We examined the data to determine whether the assessment indicators could predict the occurrence of a breach.

Because the breach is a binary variable, logistic regression was chosen as the analytical technique. In this case, the logistic regression equation is modeling the probability of a breach occurring (or not occurring) based on the ratings of the assessment indicators.

In order to perform the analysis, numbers were assigned to the color scale used in program reports. The mapping between the color scale and the scale used for analysis is as follows:

- $\quad 1=(\mathrm{G})$ green

- $\quad 2=(\mathrm{GA})$ green advisory

- $3=(\mathrm{Y})$ yellow

- $4=(\mathrm{YA})$ yellow advisory

- $5=(\mathrm{R}) \mathrm{red}^{2}$

\subsection{Results and Discussion}

Information extracted from all available MIDS-LVT DAES reports regarding the program assessment indictors is shown in Table $2 .^{3}$

2 The DAES guidance document at https://acc. dau. mil/GetAttachment.aspx?id=24422\&pname=file\&aid=2852 instructs that the advisory codes can indicate a worsening or improving status. This situation raises the possibility of yellow advisory being used to indicate a transition between green and yellow. The reading of the actual descriptions, however, indicates that yellow advisory is operationally used as the transition between yellow and red.

3 Many of the organization name designations (e.g., in column 1 of Table 2) have changed since this research was conducted. 
Table 2: DAES, DAES Assessment Indicators, and SAR Breach Data

\begin{tabular}{|c|c|c|c|c|c|c|c|c|c|c|c|c|c|c|c|c|c|c|c|c|c|c|c|c|c|c|c|c|c|c|c|}
\hline & \begin{tabular}{c|} 
Nov \\
97 \\
\end{tabular} & \begin{tabular}{|c|} 
Feb \\
98 \\
\end{tabular} & \begin{tabular}{c|} 
Nov \\
98
\end{tabular} & \begin{tabular}{c|} 
Feb \\
99 \\
\end{tabular} & \begin{tabular}{|l|} 
Jun \\
99
\end{tabular} & \begin{tabular}{c|} 
Nov \\
99
\end{tabular} & \begin{tabular}{c|} 
Feb \\
00
\end{tabular} & $\begin{array}{c}\text { Aug } \\
00\end{array}$ & \begin{tabular}{c|} 
Nov \\
00
\end{tabular} & \begin{tabular}{c|} 
Feb \\
01
\end{tabular} & \begin{tabular}{c|} 
May \\
01
\end{tabular} & \begin{tabular}{c|} 
Aug \\
01
\end{tabular} & \begin{tabular}{|c|} 
Nov \\
01
\end{tabular} & \begin{tabular}{c|} 
Feb \\
02
\end{tabular} & \begin{tabular}{|l|} 
May \\
02
\end{tabular} & \begin{tabular}{c|} 
Aug \\
02
\end{tabular} & $\begin{array}{c}\text { Nov } \\
02\end{array}$ & \begin{tabular}{c|} 
Feb \\
03
\end{tabular} & \begin{tabular}{|c|} 
May \\
03
\end{tabular} & \begin{tabular}{c|} 
Aug \\
03
\end{tabular} & \begin{tabular}{c|} 
Nov \\
03
\end{tabular} & \begin{tabular}{|c|} 
Feb \\
04 \\
\end{tabular} & \begin{tabular}{|c|} 
May \\
04
\end{tabular} & \begin{tabular}{|c|} 
Aug \\
04
\end{tabular} & \begin{tabular}{c|} 
Nov \\
04
\end{tabular} & $\begin{array}{c}\text { Feb } \\
05\end{array}$ & $\begin{array}{c}\text { May } \\
05\end{array}$ & \begin{tabular}{|c|} 
Aug \\
05
\end{tabular} & \begin{tabular}{|c|} 
Nov \\
05
\end{tabular} & \begin{tabular}{|c|} 
Feb \\
06
\end{tabular} & \begin{tabular}{|c|} 
May \\
06
\end{tabular} \\
\hline $\begin{array}{l}\text { Baselines - Original: Mar 8, } \\
1994\end{array}$ & & & & & & & & $\begin{array}{l}14 \\
\text { Jun }\end{array}$ & & & & & \begin{tabular}{|c|}
19 \\
Sep
\end{tabular} & & & \begin{tabular}{l|l}
18 \\
Jul
\end{tabular} & & & & $\begin{array}{c}14 \\
\text { Jun }\end{array}$ & & & \begin{tabular}{|c|}
13 \\
Mar \\
\end{tabular} & \begin{tabular}{|l|}
18 \\
Jun
\end{tabular} & & & & & & & \begin{tabular}{c|}
22 \\
Mar
\end{tabular} \\
\hline Breach $^{1,2}$ & Dec & $\mathbf{R}, \mathbf{P}$ & \begin{tabular}{|c|} 
Dec \\
$\mathbf{S}$
\end{tabular} & & $\mathbf{s}$ & $\begin{array}{c}\text { Dec } \\
\mathbf{S}\end{array}$ & & & & & & & \begin{tabular}{|l|} 
Dec \\
$\mathbf{R}, \mathbf{P}$
\end{tabular} & & & & $\begin{array}{c}\text { Dec } \\
\mathbf{S}\end{array}$ & & & & \begin{tabular}{|l|} 
Dec \\
S,P
\end{tabular} & & \begin{tabular}{|c|} 
Jun \\
$\mathbf{S}$
\end{tabular} & & \begin{tabular}{|c|} 
Dec \\
$\mathbf{R}$
\end{tabular} & & & & & & \\
\hline Performance Characteristics & G & G & G & G & & G & G & NR & NR & G & G & G & G & G & G & G & G & G & G & GA & GA & GA & GA & GA & GA & GA & GA & GA & GA & GA & GA \\
\hline & & & & & & & & GA & YA & NR & $\mathbf{Y}$ & NR & GA & GA & GA & $\mathbf{R}$ & YA & $\begin{array}{l}\mathbf{Y} \\
\end{array}$ & & $\mathbf{Y}$ & GA & GA & GA & GA & & GA & GA & GA & GA & GA & GA \\
\hline & & & & & & & & & & & & & $\mathbf{G}$ & $\mathbf{G}$ & & YA & $\bar{Y}$ & GA & $\bar{Y}$ & GA & GA & GA & GA & $\mathbf{G}$ & $\mathbf{G}$ & $\mathbf{G}$ & $\bar{G}$ & GA & GA & GA & $\bar{G}$ \\
\hline Test \& Evaluation & GA & GA & $\mathbf{Y}$ & $\mathbf{Y}$ & & $\mathbf{Y}$ & GA & NR & \begin{tabular}{|l|} 
NR \\
\end{tabular} & YA & YA & YA & YA & YA & $\mathbf{Y}$ & YA & $\mathbf{Y}$ & $\mathbf{Y}$ & $\mathbf{Y}$ & $\mathbf{Y}$ & GA & GA & GA & GA & GA & GA & GA & GA & GA & GA & GA \\
\hline & & & & & & & & $\mathbf{Y}$ & YA & YA & YA & YA & YA & YA & $\mathbf{Y}$ & $\mathbf{R}$ & $\mathbf{Y}$ & $\mathbf{Y}$ & $\mathbf{Y}$ & YA & $\mathbf{Y}$ & GA & GA & GA & $\mathbf{G}$ & GA & GA & GA & GA & GA & GA \\
\hline & & & & & & & & & & & & & YA & YA & \begin{tabular}{|l|} 
YA \\
\end{tabular} & $\mathbf{R}$ & $\mathbf{R}$ & YA & YA & \begin{tabular}{|l|} 
YA \\
\end{tabular} & YA & GA & GA & GA & GA & $\mathbf{G}$ & GA & GA & GA & GA & GA \\
\hline $\begin{array}{l}\text { Logistics Requirements \& } \\
\text { Readiness }\end{array}$ & G & G & GA & GA & & GA & GA & NR & NR & G & G & GA & GA & $\mathbf{R}$ & $\mathbf{R}$ & $\mathbf{R}$ & $\mathbf{R}$ & $\mathbf{R}$ & $\mathbf{R}$ & $\mathbf{R}$ & $\mathbf{Y}$ & $\mathbf{Y}$ & G & G & G & G & $\mathbf{Y}$ & & G & G & G \\
\hline & & & & & & & & & & & & & GA & $\mathbf{R}$ & $\mathbf{R}$ & $\mathbf{R}$ & $\mathbf{R}$ & $\mathbf{R}$ & $\mathbf{R}$ & $\mathbf{R}$ & $\mathbf{Y}$ & $\bar{Y}$ & $\mathbf{G}$ & $\mathbf{G}$ & $\mathbf{G}$ & $\mathbf{G}$ & $\mathbf{Y}$ & GA & $\mathbf{G}$ & $\mathbf{G}$ & $\mathbf{G}$ \\
\hline & & & & & & & & $\bar{G}$ & $\bar{G}$ & $\mathbf{G}$ & $\mathbf{G}$ & GA & & $\mathbf{R}$ & $\begin{array}{l}\mathbf{R} \\
\end{array}$ & $\mathbf{R}$ & $\begin{array}{l}\mathbf{R} \\
\end{array}$ & YA & YA & & & & & & & & & & & & \\
\hline Cost & $\mathbf{G}$ & G & $\mathbf{Y}$ & $\mathbf{Y}$ & & $\bar{Y}$ & $\bar{Y}$ & NR & \begin{tabular}{|l|} 
NR \\
\end{tabular} & $\mathbf{G}$ & $\mathbf{G}$ & $\overline{\mathbf{G}}$ & $\bar{G}$ & G & $\mathbf{G}$ & G & G & $\bar{G}$ & $\mathbf{G}$ & $\mathbf{G}$ & $\mathbf{G}$ & $\mathbf{G}$ & $\mathbf{G}$ & GA & GA & $\mathbf{G}$ & $\mathbf{G}$ & $\mathbf{G}$ & $\mathbf{G}$ & GA & GA \\
\hline & & & & & & & & NR & $\mathbf{G}$ & $\mathbf{G}$ & $\mathbf{G}$ & $\mathbf{G}$ & $\mathbf{G}$ & NR & $\overline{\mathbf{G}}$ & $\mathbf{G}$ & $\mathbf{G}$ & $\mathbf{G}$ & $\mathbf{G}$ & $\mathbf{G}$ & GA & $\mathbf{G}$ & GA & GA & GA & GA & $\mathbf{Y}$ & $\bar{Y}$ & YA & YA & GA \\
\hline & & & & & & & & & & & & & & & & & & & & & & \begin{tabular}{|l|} 
NR \\
\end{tabular} & \begin{tabular}{|l|} 
NRR \\
\end{tabular} & \begin{tabular}{|l|} 
YA \\
\end{tabular} & \begin{tabular}{|l|} 
YA \\
\end{tabular} & YA & NR & YA & $\mathbf{Y}$ & \begin{tabular}{|l|} 
NR \\
\end{tabular} & \begin{tabular}{|l|} 
NR \\
\end{tabular} \\
\hline Funding & GA & GA & $\mathbf{Y}$ & $\mathbf{R}$ & & $\mathbf{R}$ & $\mathbf{R}$ & & & $\mathbf{G}$ & $\mathbf{G}$ & $\mathbf{G}$ & $\mathbf{G}$ & GA & GA & GA & GA & $\mathbf{Y}$ & $\mathbf{Y}$ & $\mathbf{Y}$ & $\mathbf{Y}$ & $\mathbf{R}$ & $\mathbf{R}$ & $\mathbf{G}$ & $\mathbf{Y}$ & $\mathbf{G}$ & $\mathbf{G}$ & $\mathbf{G}$ & $\mathbf{G}$ & $\mathbf{G}$ & GA \\
\hline & & & & & & & & $\mathbf{R}$ & $\bar{Y}$ & $\mathbf{G}$ & $\mathbf{G}$ & $\mathbf{G}$ & $\mathbf{G}$ & $\mathbf{G}$ & $\mathbf{G}$ & GA & $\mathbf{G}$ & $\mathbf{G}$ & $\mathbf{G}$ & $\mathbf{G}$ & $\mathbf{G}$ & G & $\mathbf{G}$ & $\mathbf{G}$ & $\mathbf{R}$ & $\mathbf{G}$ & $\mathbf{G}$ & $\mathbf{G}$ & $\mathbf{G}$ & $\mathbf{G}$ & $\mathbf{G}$ \\
\hline & & & & & & & & & & & & & & & & & & & & & & & & & $\mathbf{Y}$ & & $\mathbf{G}$ & $\mathbf{G}$ & $\mathbf{G}$ & $\mathbf{G}$ & GA \\
\hline & & & & & & & & $\mathbf{R}$ & $\mathbf{Y}$ & & $\mathbf{G}$ & GA & GA & GA & GA & & GA & & $\mathrm{Y}$ & & & & & & & & & & & & \\
\hline Schedule & $\mathbf{Y}$ & $\mathbf{Y}$ & $\mathbf{R}$ & $\mathbf{R}$ & & $\mathbf{R}$ & GA & NR & \begin{tabular}{|l|} 
NR \\
\end{tabular} & $\mathbf{Y}$ & $\begin{array}{l}\mathbf{Y} \\
\end{array}$ & GA & GA & GA & GA & GA & GA & GA & $\mathbf{Y}$ & \begin{tabular}{|l|} 
YA \\
\end{tabular} & $\bar{G}$ & $\bar{Y}$ & YA & \begin{tabular}{|l|} 
YA \\
\end{tabular} & YA & $\mathbf{G}$ & $\bar{G}$ & $\bar{G}$ & $\bar{G}$ & GA & GA \\
\hline & & & & & & & & GA & GA & & $\mathbf{Y}$ & $\mathbf{Y}$ & $\mathbf{Y}$ & $\mathbf{Y}$ & GA & $\bar{Y}$ & YA & GA & $\mathbf{Y}$ & YA & $\mathbf{Y}$ & $\mathbf{Y}$ & $\mathbf{Y}$ & YA & YA & $\mathbf{G}$ & GA & $\mathbf{G}$ & $\mathbf{G}$ & GA & G \\
\hline & & & & & & & & & & $\mathbf{Y}$ & $\mathbf{Y}$ & $\mathbf{Y}$ & GA & GA & GA & $\mathbf{R}$ & $\mathbf{R}$ & $\mathbf{Y}$ & & & & & & & & & & & & & \\
\hline Contracts & $\bar{G}$ & GA & GA & $\bar{Y}$ & & YA & $\bar{Y}$ & NR & \begin{tabular}{|l|} 
NR \\
\end{tabular} & GA & $\bar{Y}$ & $\mathbf{Y}$ & $\mathbf{Y}$ & $\bar{Y}$ & GA & GA & GA & GA & $\bar{G}$ & $\bar{G}$ & $\mathbf{G}$ & $\bar{G}$ & $\mathbf{G}$ & $\bar{G}$ & $\bar{Y}$ & $\mathbf{G}$ & $\mathbf{G}$ & $\mathbf{G}$ & $\mathbf{G}$ & $\bar{G}$ & $\bar{G}$ \\
\hline & & & & & & & & & & & & & & & & & GA & GA & G & $\mathbf{Y}$ & GA & $\mathbf{Y}$ & $\mathbf{Y}$ & $\mathbf{Y}$ & $\mathbf{Y}$ & $\mathbf{G}$ & $\mathbf{G}$ & G & G & $\mathbf{G}$ & $\bar{G}$ \\
\hline & & & & & & & & YA & \begin{tabular}{|l|} 
YA \\
\end{tabular} & $\mathbf{Y}$ & $\mathbf{Y}$ & $\mathbf{Y}$ & $\mathbf{Y}$ & $\mathbf{Y}$ & GA & GA & & & & & & & & & & & & & & & \\
\hline Production & $\mathbf{G}$ & G & $\mathbf{Y}$ & $\mathbf{Y}$ & & $\mathbf{Y}$ & GA & NR & $\mathbf{G}$ & GA & $\mathbf{Y}$ & $\mathbf{Y}$ & $\mathbf{Y}$ & $\mathbf{Y}$ & GA & GA & GA & GA & $\mathbf{G}$ & $\mathbf{G}$ & $\mathbf{G}$ & $\mathbf{G}$ & $\bar{G}$ & $\mathbf{G}$ & $\mathbf{G}$ & $\mathbf{G}$ & $\mathbf{G}$ & $\mathbf{G}$ & $\mathbf{G}$ & $\mathbf{G}$ & $\bar{G}$ \\
\hline & & & & & & & & & & & & & $\mathbf{Y}$ & $\mathrm{Y}$ & GA & GA & GA & GA & $\mathbf{G}$ & $\mathbf{G}$ & $\mathbf{G}$ & $\mathbf{G}$ & $\mathbf{G}$ & $\mathbf{G}$ & $\mathbf{G}$ & $\mathbf{G}$ & $\mathbf{G}$ & $\mathbf{G}$ & $\mathbf{G}$ & $\mathbf{G}$ & $\mathbf{G}$ \\
\hline & & & & & & & & GA & $\mathbf{G}$ & GA & $\mathbf{Y}$ & $\mathbf{Y}$ & & & & & & & & & & & & & & & & & & & \\
\hline & & & & & & & & $\mathbf{R}$ & $\mathbf{Y}$ & & $\mathbf{Y}$ & $\mathbf{Y}$ & & & & & & $\mathbf{Y}$ & $\mathbf{Y}$ & & & & & & & & & & & & \\
\hline Management Structure & $\mathbf{G}$ & $\mathbf{G}$ & $\bar{G}$ & $\mathbf{Y}$ & & $\bar{G}$ & $\mathbf{G}$ & & & $\mathbf{G}$ & $\mathbf{G}$ & $\bar{G}$ & $\bar{G}$ & $\bar{G}$ & $\mathbf{G}$ & $\mathbf{G}$ & $\mathbf{G}$ & $\mathbf{G}$ & $\mathbf{G}$ & $\bar{G}$ & $\mathbf{G}$ & $\bar{G}$ & $\bar{G}$ & $\bar{G}$ & $\bar{G}$ & $\mathbf{G}$ & $\mathbf{G}$ & $\bar{G}$ & $\bar{G}$ & $\bar{G}$ & $\bar{G}$ \\
\hline & & & & & & & & & & & $\overline{\mathbf{R}}$ & GA & $\bar{G}$ & $\bar{G}$ & $\bar{G}$ & $\mathbf{G}$ & $\mathbf{G}$ & GA & $\mathbf{Y}$ & $\mathbf{Y}$ & $\bar{Y}$ & $\bar{Y}$ & $\bar{Y}$ & $\mathbf{G}$ & $\bar{G}$ & $\mathbf{G}$ & $\mathbf{G}$ & $\mathbf{G}$ & $\mathbf{G}$ & $\mathbf{G}$ & $\bar{G}$ \\
\hline Interoperability & & & & & & & & NR & NR & $\mathbf{G}$ & G & $\bar{G}$ & GA & $\mathbf{G}$ & $\bar{G}$ & $\mathbf{G}$ & $\bar{G}$ & $\bar{G}$ & $\bar{G}$ & $\bar{G}$ & $\bar{G}$ & $\bar{G}$ & $\bar{G}$ & $\bar{G}$ & $\bar{G}$ & G & $\bar{G}$ & $\bar{G}$ & $\bar{G}$ & $\bar{G}$ & $\bar{G}$ \\
\hline & & & & & & & & & & NR & GA & $\mathbf{G}$ & & $\mathbf{G}$ & GA & GA & & & & & & & & & & & & & & & \\
\hline & & & & & & & & $\bar{G}$ & $\bar{G}$ & $\bar{G}$ & $\bar{G}$ & GA & GA & GA & $\mathbf{Y}$ & $\bar{Y}$ & GA & GA & GA & & & GA & & & & & & & & & \\
\hline & & & & & & & & $\mathbf{Y}$ & YA & & & & & & & & & & & & & & & & & & & & & & \\
\hline & & & & & & & & & & & $\bar{G}$ & $\bar{G}$ & GA & $\bar{G}$ & GA & GA & GA & $\mathbf{G}$ & $\mathbf{G}$ & GA & GA & GA & GA & GA & $\mathbf{G}$ & G & $\mathbf{G}$ & $\mathbf{G}$ & $\mathbf{G}$ & $\mathbf{G}$ & $\mathbf{G}$ \\
\hline $\begin{array}{l}\text { * The DAES report data } \\
\text { month earlier than OS } \\
\text { For convenience, we } \\
9 \text { | CMU/SEI-2010-TR-024 }\end{array}$ & $\begin{array}{l}\text { (rows } \\
\text { D DAE } \\
\text { lave g }\end{array}$ & $\begin{array}{l}\text { s with } \\
\text { ES as }\end{array}$ & $\begin{array}{l}\text { bold } \\
\text { ssess }\end{array}$ & & & & $\begin{array}{l}\text { M suff } \\
\text { belo }\end{array}$ & $\begin{array}{l}\text { fixes) } \\
\text { w eac }\end{array}$ & are $u$ & & y date & & & & & & & & & & \multicolumn{11}{|c|}{$\begin{array}{ll}{ }^{1} \text { Breaches identified from SARs } \\
{ }^{2} \text { Breach Codes: } & S=\text { Schedule } \\
& R=\text { RDT\&E (Cost) } \\
P & =\text { Procurement (Cost) } \\
& N R=\text { explicitly marked as not rated }\end{array}$} \\
\hline
\end{tabular}


Nine program breach events occurred within the time period of the 31 sampled reports. ${ }^{4}$ However, there is no apparent visual relationship between any specific indicator and any breach identified by the SARS reports.

The logistic regression for using all the indicators to predict breaches is shown in Table 3.

Table 3: Combined Indicators

\begin{tabular}{lrrl}
\hline Predictor (Indicators) & \multicolumn{1}{l}{ Coef } & SE Coef & \multicolumn{1}{l}{ P } \\
\hline Constant & 79.075 & 53534.1 & 0.999 \\
\hline Performance Characteristics & -22.242 & 10483.4 & 0.998 \\
\hline Test \& Evaluation & -38.906 & 15911.3 & 0.998 \\
\hline Logistics Requirements \& Readiness & -0.566 & 1.5 & 0.717 \\
\hline Cost & -19.570 & 16374.3 & 0.999 \\
\hline Funding & 1.086 & 1.7 & 0.518 \\
\hline Schedule & -0.762 & 2.3 & 0.741 \\
\hline Contracts & 21.648 & 20869.5 & 0.999 \\
\hline Production & -2.635 & 24078.8 & 1.000 \\
\hline Interoperability & 42.520 & 39999.2 & 0.999
\end{tabular}

These results indicate no relationship (in all cases $\mathrm{P}>>0.05$, indicating no significant correlations) when using all of the available indicators together to predict the occurrence of any breach. Similarly, no significant results were found when correlating against each category of breach and against project baseline dates (the second row in Table 2).

The use of a lagged, breach-dependent variable was examined based on the notion that some amount of time might expire before effects became noticeable (a maximum of six months was considered). Since the unlagged breach variable was contemporaneous with the indicators reported quarterly, a maximum of three months could have expired.

Upon reduction of lags in the sets of independent variables (i.e., the indicators) no significant models emerged regardless of the combinations used. Individual predictors were then evaluated.

Table 4 displays the results from logistic regressions where one indicator is used to predict any breach (cost or schedule). The first column lists the indicator used. ${ }^{5}$ The second column lists the number of cases available for that particular equation. The number of cases differs due to OSD agency involvement- that is, different OSD agencies reviewed different indicators at different parts of the program life cycle. The third column lists the p-value associated with the logistic regression coefficient for that indicator. The fourth column indicates the p-value for the indicator when using the lagged breach variable. Other statistics are available from the output, but the p-values are sufficient for us to make a determination of significance. For the existence of a significant

$4 \quad$ Simultaneous breaches in multiple categories are counted as a single breach event.

5 Multiple sources provided values for each indicator but the identity of these sources is not revealed within this report. 
relationship between the indicator and the occurrence of a breach, the p-value for the coefficient must be less than 0.05 .

Notice that the use of a lagged breach variable did find that one of the funding indicators was significant in predicting a breach. We have no explanation of this occurrence and consider it an artifact to be compared with cross-program analyses.

We also examined whether these indicators could be related to the occurrence of new cost baselines, which are indicated in the second row of Table 2. Again, no significant relationships were detected.

Table 4: Isolated Indicators

\begin{tabular}{|c|c|c|c|}
\hline Indicator & \# of cases & p-value & p-value \\
\hline & & & lagged \\
\hline \multirow[t]{3}{*}{ Performance Characteristics } & 28 & 0.718 & 0.415 \\
\hline & 20 & 1.000 & 0.999 \\
\hline & 18 & 0.916 & 0.821 \\
\hline \multirow[t]{3}{*}{ Test \& Evaluation } & 28 & 0.708 & 0.186 \\
\hline & 24 & 0.431 & 0.119 \\
\hline & 19 & 0.398 & 0.669 \\
\hline \multirow[t]{3}{*}{ Logistics Requirements \& Readiness } & 27 & 0.462 & 0.830 \\
\hline & 19 & 0.465 & 0.875 \\
\hline & 11 & 0.998 & 0.348 \\
\hline \multirow[t]{3}{*}{ Cost } & 28 & 0.375 & 0.114 \\
\hline & 22 & 0.738 & 0.920 \\
\hline & 5 & 0.999 & 0.999 \\
\hline \multirow[t]{4}{*}{ Funding } & 28 & 0.167 & 0.019 \\
\hline & 24 & 0.471 & 0.386 \\
\hline & 6 & 0.998 & 0.304 \\
\hline & 9 & 0.525 & 0.855 \\
\hline \multirow[t]{3}{*}{ Schedule } & 28 & 0.114 & 0.096 \\
\hline & 23 & 0.057 & 0.592 \\
\hline & 9 & 0.578 & 0.998 \\
\hline \multirow[t]{3}{*}{ Contracts } & 28 & 0.334 & 0.193 \\
\hline & 15 & 0.123 & 0.726 \\
\hline & 9 & 1.000 & 0.999 \\
\hline \multirow[t]{2}{*}{ Production } & 29 & 0.464 & 0.747 \\
\hline & 19,19 & 0.496 & 0.414 \\
\hline (insufficient data) & $\mathrm{n} / \mathrm{a}$ & $\mathrm{n} / \mathrm{a}$ & $\mathrm{n} / \mathrm{a}$ \\
\hline (insufficient data) & $\mathrm{n} / \mathrm{a}$ & $\mathrm{n} / \mathrm{a}$ & $\mathrm{n} / \mathrm{a}$ \\
\hline
\end{tabular}




\begin{tabular}{lccc} 
Indicator & \# of cases & p-value & p-value \\
\hline Management Structure & 28,27 & 0.999 & 0.999 \\
\hline Interoperability & 21,21 & 0.930 & 0.930 \\
$\quad$ (insufficient data) & 22,22 & 1.000 & 0.999 \\
& $\mathrm{n} / \mathrm{a}, 5$ & $\mathrm{n} / \mathrm{a}$ & 0.999 \\
$\quad$ (insufficient data) & 13,12 & 0.722 & 0.194 \\
(insufficient data) & $\mathrm{n} / \mathrm{a}$ & $\mathrm{n} / \mathrm{a}$ & $\mathrm{n} / \mathrm{a}$ \\
\hline
\end{tabular}

The results in Table 5 show the lack of a significant relationship between the schedule program assessment indicator and the occurrence of schedule breaches only.

Table 5: Schedule Breaches Predicted by Schedule Indicators

\begin{tabular}{|lccc}
\hline Indicator and Sources & \# of cases & p-value & p-value \\
\hline Schedule & & & lagged \\
\hline 28 & 0.148 & 0.072 \\
23 & 0.174 & 0.416 \\
9 & 0.999 & 0.915 \\
\hline
\end{tabular}

These results confirm the visual perception that there is no significant relationship between the indicators and the occurrence of breaches. Other exploratory analyses conducted using the actual cost, cost variance, and schedule variance also showed a lack of statistically significant results. See Appendix B for further details of the analyses.

As we attempted to find evidence of systems-of-systems-related issues across a broad spectrum of programs, insurmountable problems arose and several research directions were abandoned. Extensive data mining was performed on several data sources within the DoD, such as the Joint C4I Program Assessment Tool, Cost Analysis Improvement Group (CAIG) historical database, and the DAES repository. Many of these data sources are classified, so effort was expended to obfuscate versions of classified data samples to evaluate the feasibility of research relevance. This approach proved to be very labor intensive and thus cost prohibitive.

Many of the integration support plans that were examined were unclassified and appeared to be a good source of data. It later became apparent, however, that mining meaningful data from these reports was too laborintensive and subject to compromise due to researcher bias in interpreting highly variable text-based issue statements.

It became evident that producing a large cross-sectional sample to study would be beyond our resources. We focused instead on a more in-depth study of a single program to try to find data that was easy to retrieve, of consistent quality, and relevant to our research goals. We hoped this study would give us a justification and mechanism to expand the data set across many programs. 
Similar findings were reported previously for these types of acquisition reports. In the Defense Acquisition University (DAU) technical report Acquisition Trend Metrics in the Department of Defense, the authors reviewed 34 programs whose engineering and manufacturing development phases ended between 1980 and 1996. They concluded that "given sufficient detailed milestones in program documentation, a slip in early test milestones is the best indicator of a program heading into trouble" [Swank 2000]. Consistent with our study of the MIDS-LVT program, this DAU study found that there was not sufficient information in the acquisition reports to address technical issues. "The conclusion: it is not possible to obtain leading indicators of a program problem in the technical section of the SAR. Most Acquisition Category (ACAT) I programs are so complex that not even a very good engineer analyst, not in the program office, could follow the technical evolution of the system" [Swank 2000].

Gailey, in Predictive Power for Program Success from Engineering and Manufacturing Development Performance Trends, examined a number of variables collected by the acquisition process and, for the most part, found no predictive power except in two variables [Gailey 2002]. Programs that used a "cost plus incentive fee" contract had greater success than programs using a "cost plus award fee," "fixed price incentive," or "firm fixed price" contract. Also, contracts that had no competition for the demonstration/validation phase (dem/val) had better success in engineering and manufacturing development (EMD) [Gailey 2002]. These findings correspond well with our view of the MIDS-LVT program.

Another source of DoD cost analysis studies that used SARS/DAES data is located in the various theses produced at the Air Force Institute of Technology (AFIT). In 1996, USAF Captain Gordon found no relationship between rebaselining and cost overruns [Gordon 1996]. Building on the previous studies by Sipple [Sipple 2002] and Genest [Genest 2004], Cross took a two-stage approach to statistical modeling: (1) use a logistic regression to identify whether schedule slips occurred and (2) use multiple regression models to predict the extent of schedule variance [Cross 2006]. In these models, the predictor variables accounted for $83 \%$ of the schedule variance. A more recent study by Foreman resulted in five regression models that predicted cost or schedule growth with adjusted $r^{2}$ above 0.80 [Foreman 2007]. These theses deserve more study as they use different numbers of cases derived from SARS/DAES reports and check over 80 different variables for inclusion. After qualifying their methodologies, most of these models use less than 40 cases for prediction. The reasons for so many disqualifications also point to the same factors we mention elsewhere in this report—unreported or missing data, changing definitions over time, and lack of validation.

Although significant time and effort is expended reporting and reviewing the indicators described in this report, they do not appear to have any value in predicting breaches. However, we can report that a wealth of status information is obtained by a thorough reading of the assessments and of the DAES reports. In particular, the executive summary includes a section called "Significant Developments Since Last Report" that provides a view of important developments and changes by quarter. Unfortunately, such information was not useful for predicting breaches.

The DAES/SARs reports did a thorough job of reporting many externalities that affected the MIDS program. For example, at one point, funding by one of the NATO partners was rejected by that country's parliament, necessitating a temporary bail-out by Spain and Italy to cover costs. Another example is the extensive, ongoing Program Manager (PM) discussions about planning and executing the various tests involved. The bulk of the technical discussion in DAES/SARs involved testing, which is an external factor since the products were tested by an independent DoD testing agency that had no ties to the program. 
In general, the DAES reports appear to be useful for keeping track of program issues that originate externally to the technological developments required by the project. Detailed explanations are included for many of the issues that affect program performance, with one noticeable omission: there is little information about the technology development issues of the program. Cost and contract budget data are reported, but no connections to system developments are made by the PM or Program Executive Office (PEO). 


\section{Study 3: Exploring the Extent of Programmatic Interdependence}

\subsection{Research Question}

How extensive is programmatic interdependence?

\subsection{Background}

The significance of interdependence has not been entirely lost on the acquisition community. Intuitively, program managers and acquisition staffs have understood that adding the joint aspect to any program increases the cost and complexity of programs and the systems they acquire. Awareness of the potential significance of interdependence has emerged in the form of the Defense Acquisition Executive Summary (DAES) review materials developed for every major defense acquisition program (MDAP) ${ }^{6}$. In this summary, programs are required to identify complementary programs which impinge upon or are affected by the subject programs.

\subsection{Method}

DAES interdependency charts for 2007 were examined and analyzed to identify program interdependencies. The interdependencies were graphically rendered as links between programs (nodes).

\subsection{Results and Discussion}

Figure 3 represents the program-to-program interdependencies identified by 571 MDAP programs within the DAES interdependency charts. Note that 1194 program-to-program interdependencies are identified as represented by links.

Figure 4 restricts the view to ACAT I programs only. The diagram shows 65 programs and approximately 128 interdependencies represented by the links between the nodes.

$6 \quad$ Every designated MDAP prepares an annual Defense Acquisition Executive Summary (DAES) report that is submitted to OUSD (AT\&L). Selected programs are reviewed at a quarterly DAES review. Programs so selected prepare a standard briefing package that summarizes program status and issues. Chart 5 of that package, "Interrelationships, Dependencies, and Synchronization with Complementary Systems," provides a subjective depiction of program interdependencies. 


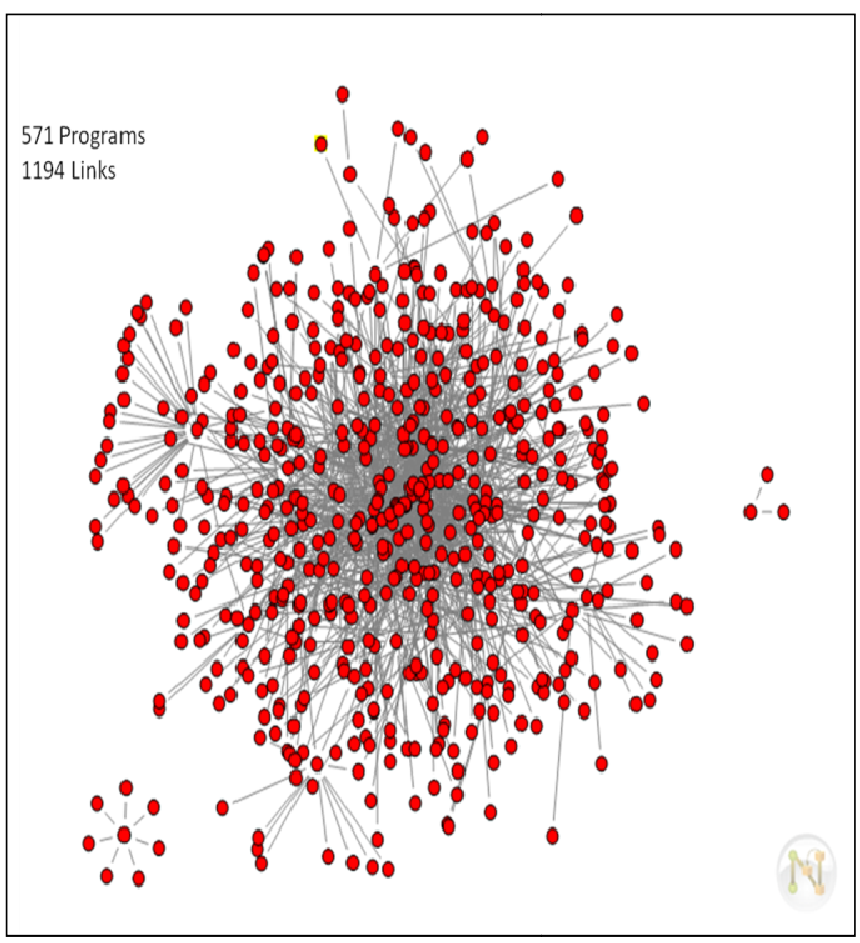

Figure 3: Mapping of Interdependencies Among all DAES Programs

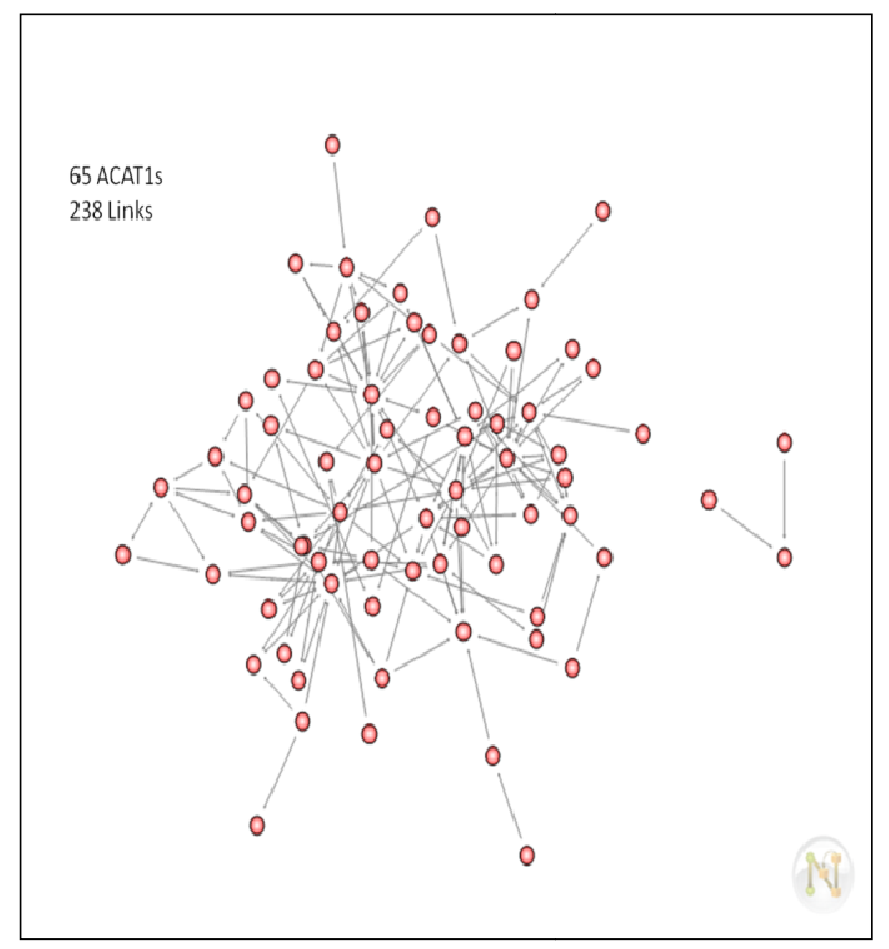

Figure 4: Mapping of Interdependencies Among ACAT I Programs 
Not surprisingly, an analysis of the DAES interdependence charts shows that DoD programs are indeed building bridges across program boundaries, with potentially significant effects with respect to interdependence.

Figure 3 and Figure 4 provide evidence that the movement to joint capabilities is well underway, and is manifested in the form of these program-to-program interdependencies. What this view does not provide, however, is insight into the potential benefits and consequences of widespread interdependence. In other words, if interdependence is widespread, does this necessarily imply a significant risk for programs that possess these interdependencies?

To investigate whether interdependence poses increased risk to programs, we looked to the DoD's long experience with joint program acquisitions, asserting that the interdependencies within joint programs represent a sub-class of interdependencies within the general set of interdependencies identified within the DAES charts. DoD programs that are explicitly identified as joint efforts ${ }^{7}$ are known to be particularly challenging. However, this recognition does not prevent these programs from exceeding cost and schedule thresholds on a routine basis. The fact that joint programs still breach more than twice as often as non-joint programs suggests that the complexity related to interdependence increases above informed prior expectations. Regardless of the precise reason for these breaches, the evidence suggests that the complexity of interdependencies — even when clearly acknowledged as a risk—appears to overwhelm current management capabilities. Given that all but a few programs are not born as "joint," systems interdependencies among the armed services guarantee interoperability and integration issues.

The pervasiveness of interdependencies also has significant implications for programs that are not explicitly identified as joint programs. As Figure 3 and Figure 4 illustrate, virtually all programs are interdependentalthough according to the official definition of joint programs, only about half are considered to be joint. ${ }^{8}$ As a consequence, programs that are presumably "non-joint" (though significantly interdependent) would experience the impact of interdependence (i.e., cost growth, schedule delay, and performance shortfalls). However, acquisition management would not anticipate these effects because without the insight into interdependence, programs thus affected are unlikely to properly attribute interdependence as a root cause to these effects. Management becomes reactive and engages in firefighting such effects as they emerge.

The Defense Acquisition Guidebook defines joint acquisition in a somewhat narrow sense. It states: "A 'joint acquisition' is any acquisition system, subsystem, component, or technology program with a strategy that includes funding by more than one DoD component during any phase of a system's life cycle" [DoD 2010].

8 Of 84 programs in 2005, 45.4\% were single-service systems and 53.6\% were joint systems [Brown 2007]. 
18 | CMU/SEI-2010-TR-024 


\section{Study 4: Using Network Methods to Explore the Evolution of Acquisition Program Interdependence Over Time}

\subsection{Research Question}

Has programmatic interdependence increased over time?

\subsection{Background}

Study 3 explored the pervasiveness of programmatic interdependence within a single year. In this study, we investigated the pattern of interdependence of programs over a 10-year span of time (1997-2007). ${ }^{9}$

Organizational theorists have long recognized that the exchange of resources (e.g., goods and services) represent a source of interdependence. Therefore, we examined the programmatic dimension of interdependence through funding exchanges between programs since we believe that these exchanges serve as effective proxies for the exchange of goods and services across program boundaries. These funding exchanges were tracked by means of the DoD program element (PE) numbers. ${ }^{10}$

\subsection{Method}

The data set used in this study was comprised of Selected Acquisition Report (SAR) information (collected during from 1997-2007) that was extracted from the Defense Acquisition Management Information Retrieval (DAMIR) system.

Funding linkages between MDAPs and program elements (PEs) were explored using network analysis tools that can examine and report characteristics of the linkages between entities (nodes).

Data for FY2000 was unavailable and therefore not included in the analysis.

10 The DoD uses six-character program element numbers in the budget process to identify each program. 


\subsection{Results and Discussion}

Table 6 lists the number of funding linkages, MDAPs, and PEs for each of the ten years of SAR data within the data set.

Table 6: Program Elements and MDAP Programs 1997-2007

\begin{tabular}{lcccccc} 
Year & $\begin{array}{l}\text { Number of } \\
\text { Linkages }\end{array}$ & $\begin{array}{l}\text { Number of } \\
\text { MDAPs }\end{array}$ & $\begin{array}{l}\text { Number of } \\
\text { Program } \\
\text { Elements (PEs) }\end{array}$ & $\begin{array}{l}\text { Ratio of PEs } \\
\text { per MDAP }\end{array}$ & $\begin{array}{l}\text { Number of } \\
\text { Links per } \\
\text { MDAP }\end{array}$ & $\begin{array}{l}\text { Number of Links } \\
\text { per PE }\end{array}$ \\
\hline 1997 & 82 & 24 & 80 & 3.3 & 3.4 & 1.03 \\
\hline 1998 & 96 & 25 & 92 & 3.5 & 3.7 & 1.04 \\
\hline 1999 & 87 & 26 & 84 & 3.2 & 3.3 & 1.04 \\
\hline 2000 & & & Data unavailable for FY2000 & & \\
\hline 2001 & 113 & 31 & 104 & 3.4 & 3.6 & 1.09 \\
\hline 2002 & 116 & 35 & 105 & 3.0 & 3.3 & 1.10 \\
\hline 2003 & 117 & 37 & 106 & 2.9 & 3.2 & 1.10 \\
\hline 2004 & 135 & 44 & 120 & 2.7 & 3.1 & 1.13 \\
\hline 2005 & 159 & 50 & 135 & 2.7 & 3.2 & 1.18 \\
\hline 2006 & 257 & 92 & 218 & 2.4 & 2.8 & 1.18 \\
\hline 2007 & 319 & 95 & 257 & 2.7 & 3.4 & 1.24 \\
\hline
\end{tabular}

Evidence of increasing programmatic interdependence is revealed in Figure 5, which depicts the number of linkages per PE over time. Throughout its life cycle, each MDAP will exchange funding with each of the PEs that it is associated with, including: (1) Research, Development, Test, \& Evaluation (RDT\&E), (2) Procurement, and (3) Operating and Maintenance (O\&M) appropriations. However, the number of funding links per MDAP is greater than three (for each year of its life cycle). This suggests that each MDAP is exchanging resources with another $\mathrm{PE}$ at some time in its life cycle.

This dynamic is also reflected by the number of MDAPs that receive funds from each PE. Each PE is tied to a particular appropriation (e.g., RDT\&E, Procurement, O\&M), and each PE will have principally one program that it supports. However, the average number of MDAPs linked to a given PE has grown from 1.03 to 1.24. This suggests that PEs are increasingly providing resources to more than their primary programs.

Why would such exchanges take place? One potential explanation is that these links reflect exchanges of goods and services across MDAP boundaries. Therefore the existence of these funding exchanges reflects the existence of program-to-program interdependencies. 


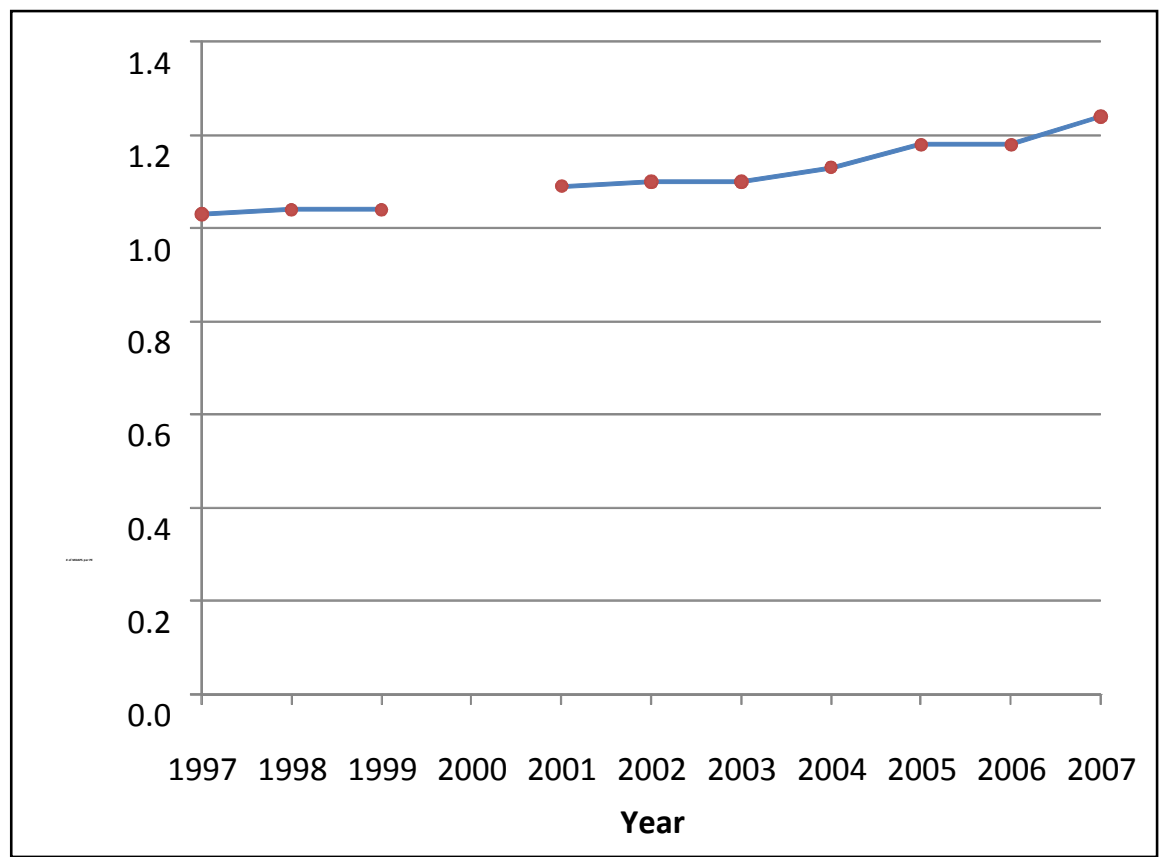

Figure 5: Number of MDAPs per Program Element from 1997 to 2007

Figure 6 shows the pattern of resource exchanges (links) between PEs and MDAPs as of 1997. Note the two clusters that are revealed in the diagram. Both clusters show that only two MDAPs share common program elements in 1997. However, by 2007, the relationships had grown increasingly complex, as illustrated in Figure 7. The two original clusters remained essentially the same, but the number of other clusters, and their associated link densities, increased dramatically. 


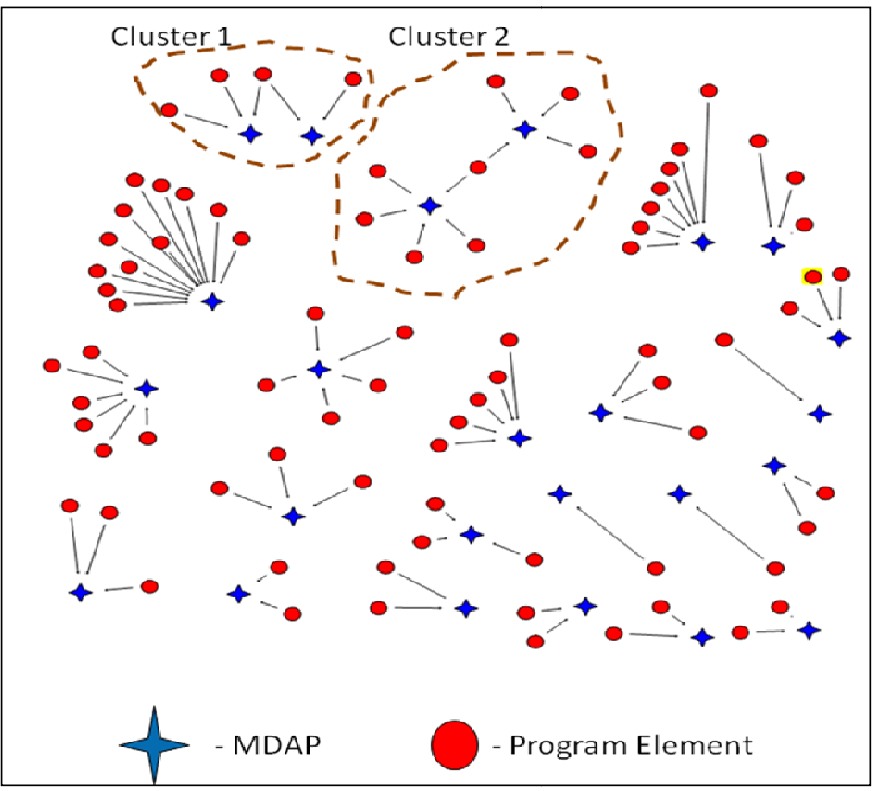

Figure 6: Program Clusters as of 1997

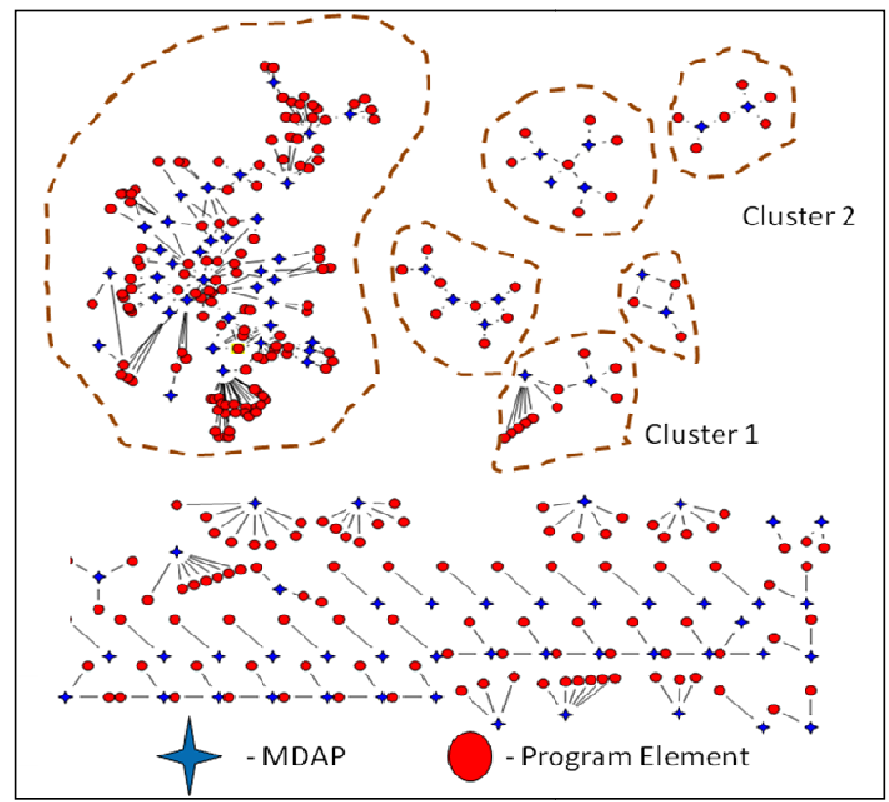

Figure 7: Program Clusters as of 2007 
The average degree for a network is defined as the sum total of all node linkages divided by the number of nodes within the network. For example, in Figure 8, Node A has three links, Node B has four links, Node C has one link, Node D has one link, Node E has two links, and Node F has three links. Therefore, the average degree $(\mathrm{AD})$ is defined as:

$A D=\frac{\text { Total Node Links }}{\# \text { of Nodes }}=\frac{3+4+1+1+2+3}{6}=\frac{14}{6}=2.3$

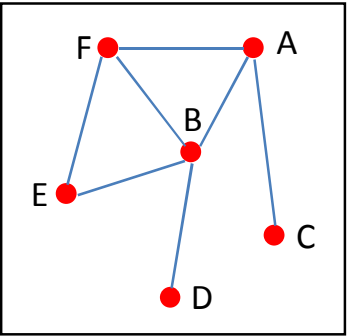

Figure 8: Example-Calculating Average Degree

As illustrated in Figure 9 the average degree for PE to MDAP relationships grew from 0.2 during 1997 to 0.78 during 2007—an approximate four-fold increase. This increase occurred even though the average number of PEs per MDAP did not change over the 10-year timeframe.

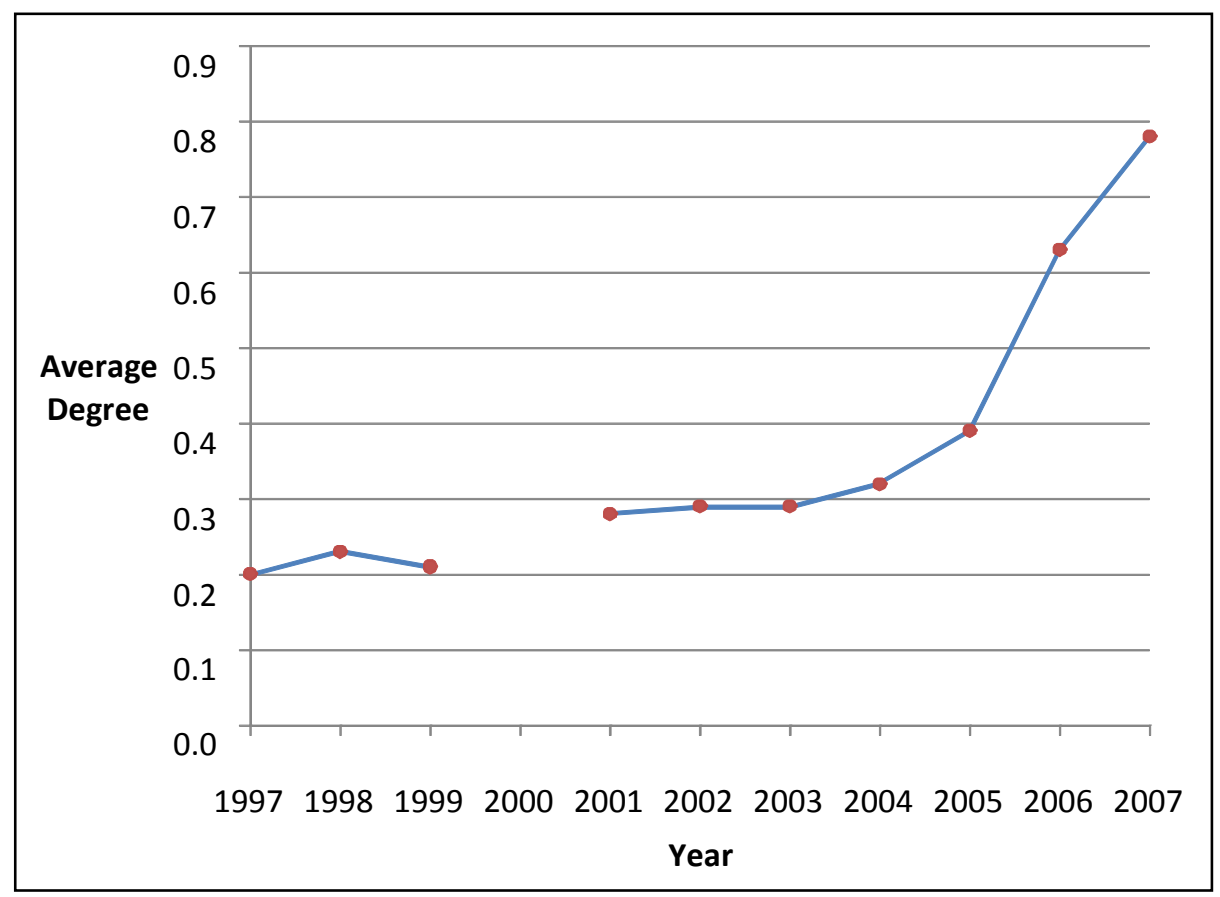

Figure 9: Average Program Network Degree by Year

The growth in the way the MDAPs interconnect through their respective PEs is not surprising given the movement toward joint capabilities. The network diagrams support the hypothesis that the complexity of 
acquisition efforts has grown along with increasing emphasis on supporting joint capabilities. Unfortunately this increasing complexity is hidden by the program-centric nature of the SAR—only by compiling individual observations is this mosaic of interdependencies and corresponding complexity revealed.

The increasing complexity of the relationships is hidden by the program-centric nature of the SAR. However, by compiling individual MDAP-PE relationships, this mosaic of interdependencies and corresponding complexity is revealed. 


\section{Study 5: Using Network Methods to Explore the Relationship Between Systems Development Interdependencies and Development Resources}

\subsection{Research Question}

What is the relationship between constructive interdependence and a program's development resource demand?

\subsection{Background}

Constructive interdependencies are a result of the technical requirements that drive the development of a complex system to meet the needs of the end user (that is, the operational domain). In more customary terms for the DoD acquisition domain, the constructive dimension addresses the engineering of systems- the construction of system components and the integration of these components into a coherent whole. The artifacts developed within the constructive domain are systems engineering artifacts including the architectural depictions that describe the top-level components of the system and the relationships between the components.

Recalling our fundamental research premise that interdependence affects the effort (and thus the cost) of developing systems, the artifacts most likely to reveal these characteristics are those that explicitly describe interdependencies. The Information Support Plan (ISP) is such an artifact. The ISP requires the program to describe the information support requirements the system in question receives from and provides to external entities.

The interdependencies described in the ISP are limited to information flows. They do not describe such things as physical interfaces, electrical power requirements, air conditioning, or other aspects of constructive interdependencies. Furthermore the ISP describes these information flows from the perspective of the system itself, rather than a more global treatment of information flows. Nevertheless, the ISP still addresses interdependence in a meaningful and consistent way.

With the advent of the DoD Architecture Framework (DoDAF), a standard lexicon and semantics for describing constructive interdependence has made the ISP a document of unique significance and increasing utility for the analysis of constructive interdependence. The DoDAF describes entities and relationships through a number of "views" which are designed to convey information in a way that is relevant to a particular constituency in terms that are understandable in that domain. ${ }^{11}$

One of the principal benefits of using an established architecture framework like DoDAF is to enforce linkages between the operational and the constructive domains of interdependence. As such, the resulting integrated architecture is in effect a systems engineering product that relates operational requirements to system design attributes.

It should be noted that the consistency and quality of the DoDAF artifacts constructed by DoD programs reflects the evolving nature of architecture as a discipline, and therefore the programs with the best ISP artifacts are those that have not yet completed development. For these programs, the full story of development

11 The views are characterized as AV (All View), SV (Systems View), OV (Operational View), and TV (Technical View), with a numerical designator that corresponds generally to the level of detail revealed in that view [DoDAF 2007]. 
cost growth has not been told; therefore, this analysis method does not yet address critical issues such as propensity for cost or schedule growth relative to the milestone $\mathrm{B}$ baseline. This deficiency will resolve itself in due course, as programs "age into" milestone C, the development budgets will become sunk costs, and at that point, this method will be able to address development cost growth, schedule delay, and baseline breaches.

\subsection{Method}

In this study, we used the Operational View 2 (Operational Node Connectivity Description) and the Systems View 6 (Information Exchange Matrix), as defined by DoD Architecture Framework Version 1.5 [DoDAF 2007].

\subsubsection{Measuring Constructive Interdependence}

In this study, constructive interdependencies are characterized as information flows between elements of the MDAP systems under construction. We employed network analysis techniques to analyze the information provided by the SV-6 DoDAF data. Therefore, we equate the term "node" for a program entity and "link" to represent information flow. The use of the terms "links and nodes" does not suggest a particular implementation of information flow. ${ }^{12}$ The means of information flow are determined by a specific program and vary considerably.

For this study, characteristics of constructive interdependence are described in Table 7.

\section{Table 7: $\quad$ Description of Network Terms Used for Analysis}

\begin{tabular}{lcl} 
Item & Symbol & Description \\
Node & $\mathrm{N}$ & $\begin{array}{l}\text { An architecture element that produces, } \\
\text { consumes, or processes data. }\end{array}$ \\
\hline Send node & $\mathrm{N}_{\mathrm{s}}$ & A node that sends information \\
\hline Receive node & $\mathrm{N}_{\mathrm{r}}$ & A node that receives information \\
\hline Send/receive node & $\mathrm{N}_{\mathrm{s} / \mathrm{r}}$ & A node that sends and receives information \\
\hline Total nodes & $\mathrm{N}_{\mathrm{T}}$ & Total number of nodes within the system \\
\hline Link & $\mathrm{L}$ & $\begin{array}{l}\text { A physical or logical connection between } \\
\text { nodes }\end{array}$ \\
\hline Uni-directional link & $\mathrm{L}_{\mathrm{dd}}$ & A link with a uni-directional information flow \\
\hline Bi-directional link & $\mathrm{L}_{\mathrm{bd}}$ & Link with a bi-directional information flow \\
\hline Total links & $\mathrm{L}_{\mathrm{T}}$ & Total number of links in the system \\
\hline Maximum links & $\mathrm{L}_{\mathrm{Max}}$ & Maximum number of links that are possible \\
\hline Links per node & $\mathrm{L}_{\mathrm{T}} / \mathrm{N}_{\mathrm{T}}$ & $\begin{array}{l}\text { Total number of links divided by total } \\
\text { number of nodes }\end{array}$ \\
\hline
\end{tabular}

12 The basis for the definitions of nodes and links is the DoD Architecture Framework Version 1.5 Volume I: Definitions and Guidelines [DoDAF 2007].

13 The maximum possible links is computed according to Metcalfe's Law, which states that the maximum number of possible linkages in a network is derived from the formula LMax $=N T^{*}(N T-1) / 2$ [Metcalfe 1995]. 


\begin{tabular}{lcl}
\hline Item & Symbol & Description \\
\hline Integration density & $\mathrm{L}_{\mathrm{T}} / \mathrm{L}_{\mathrm{Max}}$ & $\begin{array}{l}\text { Total number of links divided by the } \\
\text { maximum number of links that are possible }\end{array}$ \\
\hline
\end{tabular}

To quantitatively assess the number of nodes and links within a system, each node (entity) was counted once and each link (relationship) was counted once regardless of how many times the entity (node) or relationship (link) was invoked within the DoDAF SV-6. Therefore, the measure of interdependence used within this study is based on unique links and nodes. Figure 10 provides an example to illustrate the counting rules that were used within this analysis.

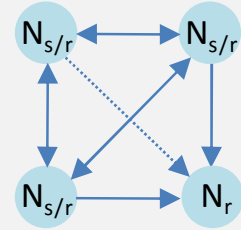

- Actual link
Potential link

\begin{tabular}{|c|c|c|c|}
\hline & Item & Symbol & Value \\
\hline \multirow{4}{*}{$\begin{array}{l}\text { y } \\
\frac{d}{0} \\
z\end{array}$} & Send/Receive & $\mathrm{N}_{\mathrm{s} / \mathrm{r}}$ & 3 \\
\hline & Send & $\mathrm{N}_{\mathrm{s}}$ & 0 \\
\hline & Receive & $\mathrm{N}_{\mathrm{r}}$ & 1 \\
\hline & Total & $\mathrm{N}_{\mathrm{t}}$ & 4 \\
\hline \multirow{6}{*}{$\stackrel{\tilde{\check{L}}}{\underline{\Xi}}$} & Uni-directional & $\mathrm{L}_{\mathrm{ud}}$ & 2 \\
\hline & Bi-directional & $\mathrm{L}_{b d}$ & 3 \\
\hline & Total & $\mathrm{L}_{\mathrm{t}}$ & 5 \\
\hline & Metcalfe number & $L_{t \text { Max }}$ & 6 \\
\hline & Integration density & $\mathrm{L}_{\mathrm{t}} / \mathrm{L}_{\mathrm{tMax}}$ & $5 / 6$ \\
\hline & Links per node & $\mathrm{L}_{\mathrm{t}} / \mathrm{N}_{\mathrm{t}}$ & $5 / 4$ \\
\hline
\end{tabular}

Figure 10: Example: Node and Link Counting Rules Applied to ISP Documents

\subsubsection{Measuring Development Resource Demand}

For this study, development resource demand is defined as the Research, Development, Test, and Evaluation (RDT\&E) program budget.

In this study, the number of links and nodes represented the independent variable that we used as a proxy measure for interdependence. The dependent variable is development resource demand.

\subsection{Results and Discussion}

\subsubsection{Part I - Relationship between Total Number of System Nodes and Integration Density}

As we applied the counting rules to the ISP data for the MDAPs in our sample, we discovered a useful pattern in the relationship between the total number of system nodes $\left(\mathrm{N}_{\mathrm{T}}\right)$ and the integration density $\left(\mathrm{L}_{\mathrm{T}} / \mathrm{L}_{\mathrm{Max}}\right)$. This pattern is illustrated in Figure 11. The pattern was consistent across a wide range of system sizes and a diversity of MDAPs that were included in the data set. 
The consistency of the pattern provided the ability to address variability in the way the SV-6 DoDAF data was interpreted from program to program and to objectively address many of the apparent inconsistencies among the documents. For example, if a program suggested that it either had significantly greater or fewer links for a given size (that is, total number of nodes), a review of the supporting documentation would typically reveal some fundamental error within the ISP documentation. Likewise, we noted that progressive programs that used architecture tools tended to have more consistent and reliable ISP products.

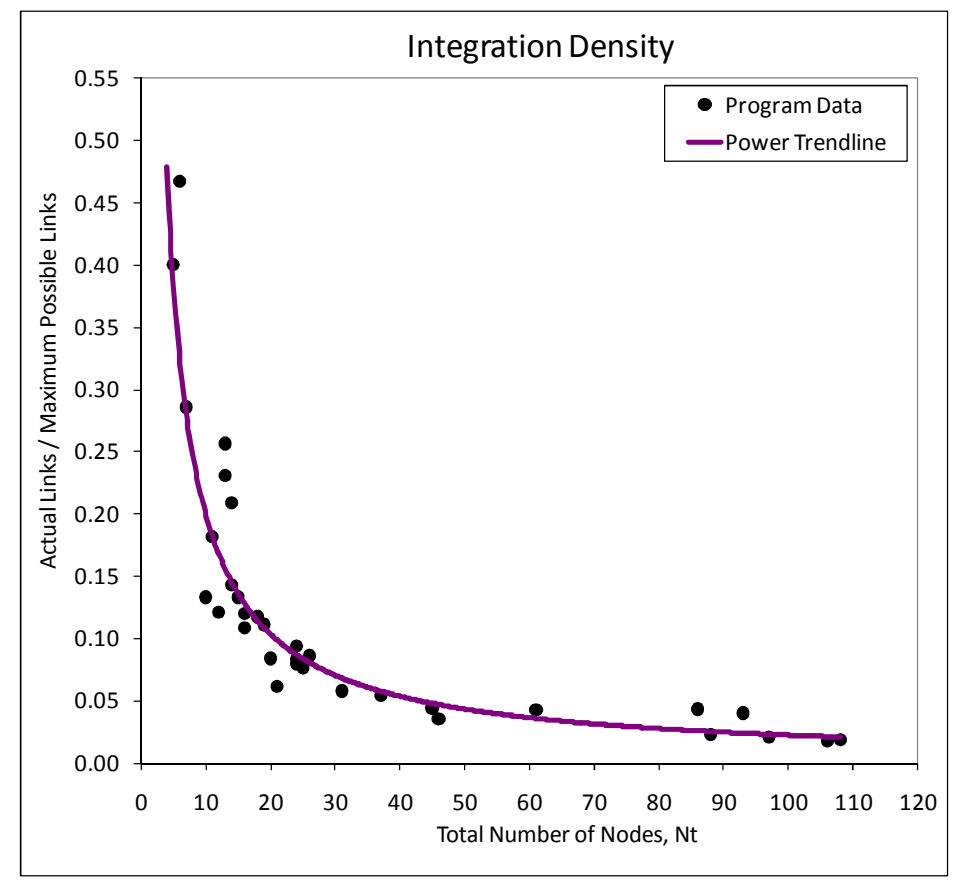

Figure 11: Integration Density of MDAPs

Beyond using this relationship to validate extracted DoDAF data, the relationship illustrated in Figure 11 also provides a way to estimate the number of links that a program with a given number of nodes would have. Thus, the number of program nodes or links can be used as a proxy measure for system size.

\subsubsection{Part II - Relationship Between Constructive Interdependence and Development Resource Demand}

If the effect of complexity and interdependence is to increase development effort, we would expect to see a positive correlation between the measures of interdependence and the corresponding RDT\&E budgets. Stated more formally in terms of the data: according to our hypothesis, RDT\&E resources are influenced by three factors:

1. Number of nodes and links

2. Node and link complexity

3. Interdependence-related complexity (measured in number of links per node)

We observed a positive, non-linear correlation between the number of nodes and development resource demand as measured by RDT\&E budget (see Figure 12) and also between the number of links and RDT\&E budget (see Figure 13). As size (number of nodes) and interdependence (number of links) increase, the amount of development resources required increases according to a power-law relationship. 


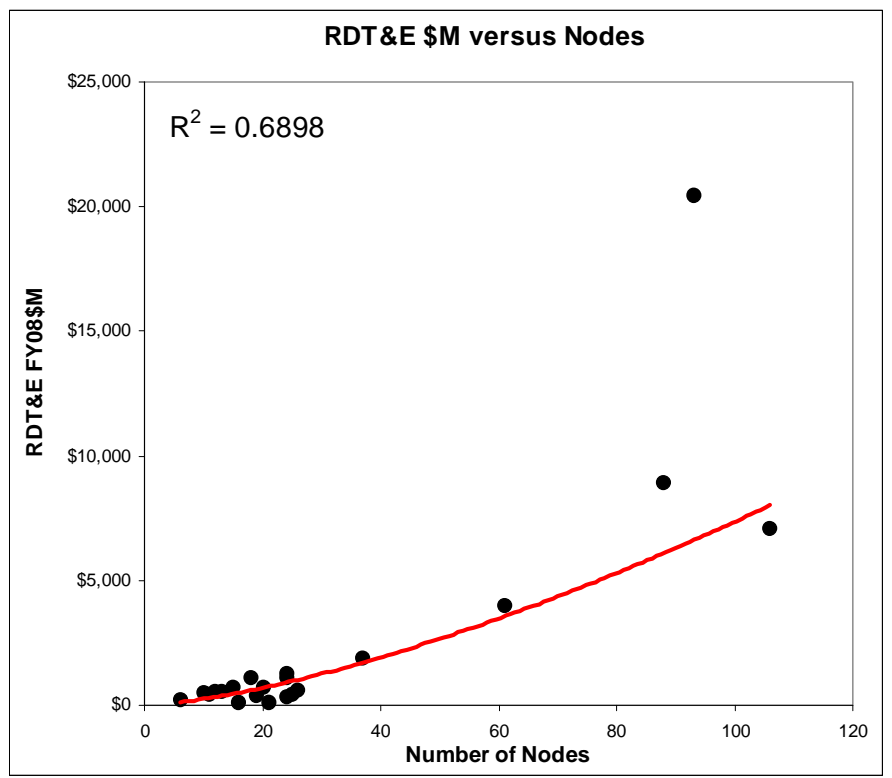

Figure 12: Development Resource Demand Versus Number of Nodes

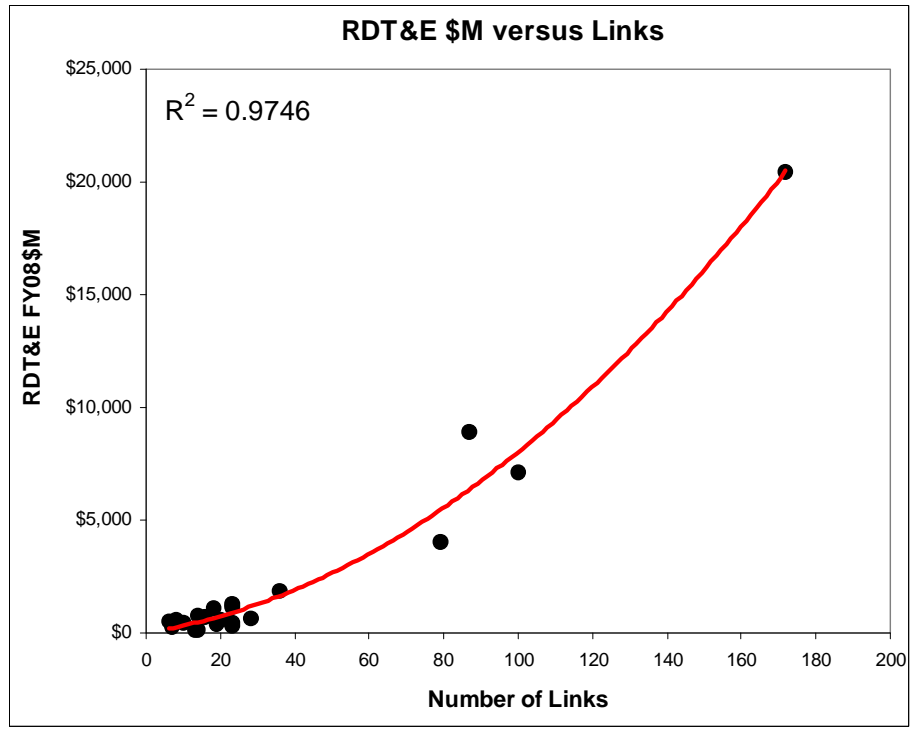

Figure 13: Development Resource Demand Versus Number of Links 


\subsubsection{Part III - Development of the Equivalent Node Measure}

To explore the relationship between nodes, links, and development resource demand, a model was proposed that incorporates the combined effects of size and interdependence. To develop this model, we drew a conceptual analogy between the software cost analysis approach of counting Equivalent Source Lines of Code (ESLOC) based on a weighted combination of new, reused, and modified software lines of code and then using this value as input to a size-driven parametric software cost models. ${ }^{14}$

In our case, we explored the notion of an equivalent node (which is a weighted combination of send-only, receive-only, and send-receive nodes) and an interdependence-driven complexity factor represented by the normalized number of links per node. Our proposed model captures the following characteristics:

1. Send/Receive nodes are more complex than Send-Only or Receive-Only nodes

2. Send-Only nodes are more complex than Receive-Only nodes

3. Nodes that have more links are more complex than nodes with fewer links

The resulting formulation is expressed by Equation 1.

$$
N_{e}=\left(d N s / r+g N_{s}+h N_{r}\right)\left(\frac{L_{t} / N_{t}}{\operatorname{avg}\left(L_{t} / N_{t}\right)}\right)^{c}
$$

where:
$N_{e} \quad$ is the equivalent nodes value
$N_{S} \quad$ is the number of send-only nodes for the program
$N_{r} \quad$ is the number of receive-only nodes for the program
$\mathrm{Ns} / \mathrm{r} \quad$ is the number of send-receive nodes for the program
$L_{t} \quad$ is the total number of links for the program
$N_{t} \quad$ is the total number of nodes for the program
$d \quad$ is the derived coefficient reflecting the relative weight of the send-receive nodes
$g \quad$ is the derived coefficient reflecting the relative weight of the send-only nodes
$h \quad$ is the derived coefficient reflecting the relative weight of the receive-only nodes
$c \quad$ is the derived exponent that scales node complexity according to the interdependence-driven complexity of the program

14 Most parametric software cost models use a size-driven algorithm to compute cost, effort, and schedule. In order to adjust these models to predict the cost of software that is reused or modified rather than designed from scratch, various weighting schemes have been developed. These weighting methods typically assign newly-developed code a weighting factor of 1.0 , with reused or modified code having some fraction of the new code weight. The weights calculate the amount of equivalent new software that would be developed to have the same cost as the modified and reused code. Thus, an "equivalent" source line of code is input into the conventional parametric model. 
Once the value of equivalent nodes $\left(N_{e}\right)$ is computed, the relationship between equivalent nodes and development resource demand can be expressed as in Equation 2.

$$
R D T \& E \$=a N_{e}^{b}
$$

where:

$$
\begin{aligned}
& R D T \& E \$ \text { is the development resource demand, monetized as research, development, test, and } \\
& \text { evaluation dollars expressed in fiscal year } 2008 \text { constant dollars X } 10^{6} \\
& \text { a is the derived scaling coeffient } \\
& N_{e} \quad \text { is the value for equivalent nodes (calculated by Equation 1) } \\
& b \quad \text { is the derived exponential scaling factor }
\end{aligned}
$$

Having proposed a functional form for equivalent nodes $\left(N_{e}\right)$ and development resource demand (RDT\&E $\$$ ), the values of the various weight factors and coefficients are derived using the ISP data and an optimization routine (Microsoft Excel Solver) that minimizes the residuals for a nonlinear equation relating nodes to resource demand (millions of RDT\&E dollars).

The results of the optimization routine lead to the following expression:

$$
N_{e}=\left(N s / r+0.5 N_{s}+0.29 N_{r}\right)\left(\frac{L_{t} / N_{t}}{1.02}\right)^{1.22}
$$

where we find that

$$
\begin{aligned}
& d \quad \text { the relative weight of send/receive nodes, is set to } 1.0 \text { (by definition) } \\
& g \text { the relative weight of send-only nodes is } 0.5 \text {, which implies that send-only nodes are } \\
& \text { half as complex as send/receive nodes } \\
& h \text { the relative weight of receive-only nodes is } 0.29 \text {, meaning that receive-only nodes are } \\
& 29 \% \text { as complex as send/receive nodes } \\
& \operatorname{avg}\left(L_{t} / N_{t}\right) \text { the interdependence-driven complexity scaling factor is } 1.22 \text {, meaning that node } \\
& \text { complexity has a positive, non-linear effect on development resource demand }
\end{aligned}
$$

Deriving the parameters of the basic nonlinear equation relating equivalent nodes to overall development resource demand (Equation 2, above) results in the following expression:

$$
R D T \& E \$=20.7 N_{e}^{1.38}
$$

where we find that

a the derived scaling coefficient, is $\$ 20.7\left(\mathrm{X}^{6} 0^{6}\right)$

$b \quad$ the derived exponential scaling factor, is 1.38

As illustrated in Figure 14, the relationship expressed in Equation 4 demonstrates a remarkable fit to the data set, demonstrating a coefficient of correlation of 99 percent. 


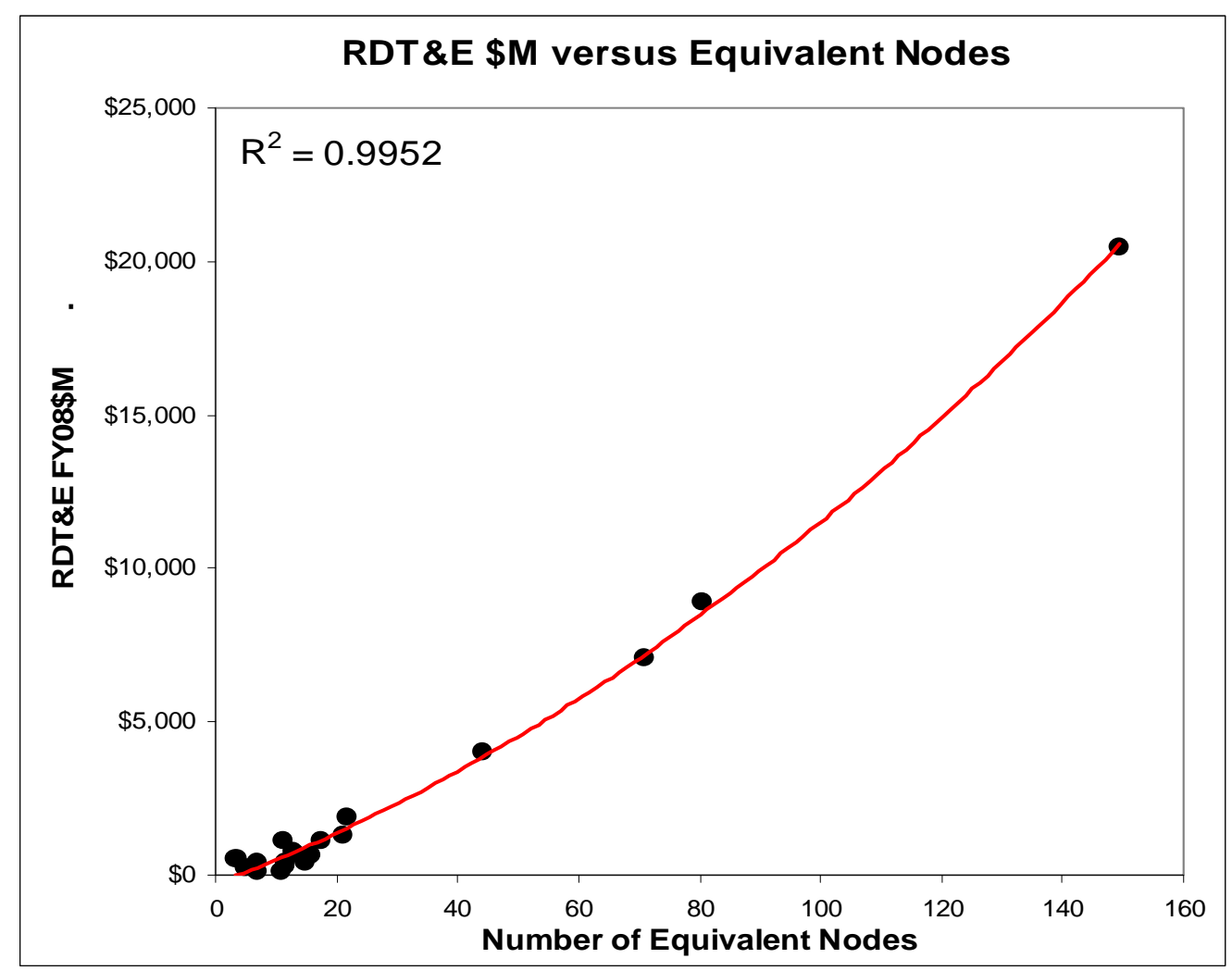

Figure 14: Fitted Equation of Equivalent Nodes to Development Resource Demand

The utility of the relationship expressed in Figure 14 is significant for assessing the approximate level of development resource demand for programs of a given size and level of interdependence. Measures of constructive interdependence can be assessed from standard documents prepared relatively early in the program lifecycle. Analyzing the degree of interdependence early in the program planning process will improve estimates of development budgets to ensure that adequate resources are made available to support systems engineering, architecture development, and risk mitigation activities during system development. This may reduce the incidence of program overrun since the previously hidden effects of interdependence-driven complexity can now be explicitly described and their effects on cost escalation predicted. However, this will also raise the apparent cost of programs early in the program lifecycle and make some programs appear unaffordable. Therefore, such assessments may be met with skepticism or outright hostility. When this is the case, the tendency might be to defer such assessments to later phases in the development lifecycle so that these additional costs and impact to schedule are underemphasized. Unfortunately, the history of software and systems engineering programs is replete with examples of such a strategy's enormous impact on cost growth or a dramatic reduction of technical performance.

The results of this study are interesting when combined with the results of Study 4. Figure 6 and Figure 7 show evidence of programs sharing resources across their boundaries and creating increased financial (programmatic) interdependencies as a result. Figure 13 and Figure 14 indicate significant increases in development cost demand with increasing interdependence. This may suggest that programs facing overwhelming complexity and associated costs are searching out ways to offset the cost of this complexity and are doing so by creating collaborative programmatic clusters to work through shared challenges. Such 
collaboration, while aligned with the DoD leadership's promotion of a joint doctrine, no doubt faces substantial governance barriers structured by the existing service-centric and program-centric paradigm.

The observed power law relationship between nodes and their links also suggests an underlying innate phenomenon may be at work. In naturally-occurring networks, growth occurs by establishing links among nodes in the most cost-effective way possible: by providing access to the greatest number of resources through the fewest possible links. Under such circumstances, the law of preferential attachment emerges which states that when adding nodes to a network, some nodes are more attractive targets for links than others. These nodes may themselves have a richer set of resources or may be better-connected than others. Therefore, they are able to indirectly provide access to a wider range of resources. These well-connected nodes tend to attract yet more connections, becoming highly-connected "hubs." In this way, through the natural behavior of each node making local optimizing decisions during network growth, some nodes may develop many links while the majority of nodes might have only one. This gives rise to a links per node distribution that follows a power law relationship similar to that observed in our data set.

Such networks have been called "scale-free" and manifest interesting and useful characteristics that in the future may help explain and predict the behaviors of the defense acquisition enterprise [Laszlo-Barabasi 2002, pp. 86-87]. However, in the near-term, even these early and relatively simple insights provide a significant increase in descriptive and predictive power over traditional program-centric management approaches. Additional research in these areas should prove extremely fruitful. 
34 | CMU/SEI-2010-TR-024 


\section{Summary}

This report described five studies that were part of a major research initiative sponsored by the Office of the Deputy Under Secretary of Defense (Acquisition and Technology) Systems and Software Engineering / Strategic Initiatives.

The overall goal of the research was to identify, quantify, and assess the degree of interdependence and to assess the effects of that interdependence on program risk.

Throughout all of these studies, the researchers attempted to identify representations within data constructs that would capture the phenomenon of interdependency. However, there is a need to validate the effectiveness of the measures through additional studies. Generally speaking, we do not want to assert that the approaches used in our studies represent the only ways to characterize interdependency. The identification of effective ways of measuring interdependency is a topic that would benefit from additional research.

Our research approach began with Study 1 that explored the qualitative factors that confound program cost and schedule estimation. Study 2 employed data-mining and statistical analyses to determine whether DAESSARS information can be used to forecast program performance. An interesting result from this study is that there was no evidence that such indicators are effective in predicting program breaches. Studies 3 through 5 employed network analysis techniques to quantitatively characterize programmatic and constructive interdependences in the acquisition enterprise. These last three studies culminated in graphical models that related interdependence and program cost.

In this paper, we reported a number of important findings and noteworthy insights that are available when considering programs in light of their interdependencies with other programs. In particular, four critical findings were revealed by this research.

First, our research study found no evidence that indicators reported within Defense Acquisition Executive Summary (DAES) reports or Select Acquisition Reports (SARs) predict program breach events.

Second, limiting the definition of interdependence to programs that are identified and funded as joint programs is insufficient. All programs are interdependent to some degree, and therefore interdependence-related risk is widespread, regardless of service affiliation or participation. The examination of individual program characteristics in isolation from other programs falls short of the mark for understanding risks to the program, particularly those risks arising from interdependencies. An expanded definition of interdependencies, based on rigorous investigation, is likely to provide further insights into program risk.

Third, even the relatively aggregated SARs data and the often-disparaged DoDAF artifacts, when combined in an analytically rigorous manner over a sufficiently large sample, can provide significant insights into program behaviors that are unavailable elsewhere. Continued investment in the gathering of objective, authoritative data should be a major emphasis across the DoD. The consequence of failing to gather and analyze these important data is substantial program failure, wasted resources, and erosion of the public trust in the integrity and capability of the DoD. 
Finally, traditional methods of analyzing risk, while important, need to be supplemented with network analysis techniques to reveal the true scope and effects of programmatic and constructive interdependence. Additional investigation into methods and measures that can reveal critical interdependencies is clearly warranted.

These results indicate that an expanded definition of interdependencies along with the incorporation of network analysis tools may provide important insights into program performance in a joint capability arena. It is, thus, an important topic of inquiry. 


\section{Appendix A: Diagnostic Risk Indicators Associated with Integration and Interoperability}

\section{Overview}

This appendix presents the results of Study 1, which is described on page 5.

\section{Missing Requirements}

Missing requirements are a significant source of estimation error and cost variance related to interoperability and integration (I\&I) efforts. Although missing requirements have always troubled complex software systems, the issue escalates with each dimension of I\&I complexity: systems, services, knowledge domains, funding sources, users, stakeholders, and interfaces.

1. What evidence exists that the program has an understanding of the complexity of the problem space, the solution space, and the required software?

\begin{tabular}{lllll}
\hline Architectural views & Operational & $(+)$ & System & $(+)$ \\
& Technical & $(+)$ & Cross-correlated $^{15}$ & $(++)$ \\
& Software & $(+)$ & None & $(-)$ \\
\hline Scenario thread analysis & Extensive & $(+)$ & Cross domains & $(+)$ \\
& None & $(-)$ & & \\
\hline Use cases & Extensive & $(+)$ & User-validated & $(+)$ \\
& None & $(-)$ & & \\
\hline Stakeholder involvement & Continuous & $(+)$ & Comprehensive & $(+)$ \\
& Measured, common, & $(+)$ & None & $(-)$ \\
& shared understanding & & & $(+)$ \\
\hline Simulation efforts & Extensive & $(++)$ & Sparse & \\
\hline Fragmented understanding & Measured assessment & $(+)$ & No evaluation & $(-)$ \\
\hline
\end{tabular}

15 The DoDAF views do not typically represent software architectures; therefore, a mapping of the DoDAF views into software architectures should be evidenced. 
2. Requirements volatility is considered to be a major source of risk to the management of large and complex software projects.

\begin{tabular}{|c|c|c|}
\hline \multirow[t]{4}{*}{ Team conflict } & Minimalist reduction solutions (LCD) & $(+)$ \\
\hline & Optimized union of stakeholder demands & $(++)$ \\
\hline & Measured, common, shared understanding & $(+)$ \\
\hline & Clear prioritization of requirements & $(+)$ \\
\hline \multirow[t]{7}{*}{ Volatility management } & Claims of stability ${ }^{16}$ & $(-)$ \\
\hline & Volatility acknowledged & $(+)$ \\
\hline & Process to monitor & $(+)$ \\
\hline & Formal change management & $(+)$ \\
\hline & Configuration control & $(+)$ \\
\hline & Quality Management processes & $(+)$ \\
\hline & Nature and origins of requirements change analysis & $(+)$ \\
\hline \multirow[t]{4}{*}{ Data models } & Simplistic "post all data to shared space"17 & $(-)$ \\
\hline & Holistic DOTMLPF ${ }^{17}$ examination & $(+)$ \\
\hline & Full mission thread simulations & $(+)$ \\
\hline & Scenario-based vetting of the system complexities & $(+)$ \\
\hline \multirow[t]{5}{*}{ Scope creep } & Problem solving process elicits critical requirements & $(+)$ \\
\hline & Precisely articulated requirements & $(+)$ \\
\hline & Opportunistic goal-seeking behaviour & $(-)$ \\
\hline & $\begin{array}{l}\text { Controls for unexpected changes in the operational } \\
\text { environmental context }\end{array}$ & $(+)$ \\
\hline & Evidence of shared understanding and consensus & $(+)$ \\
\hline
\end{tabular}
volatile requirements.

17 Making data available should not be accompanied by an assumption of utilization. It takes Doctrine, Operations, Logistics, Training, Leadership, Personnel, and Facilities (DOT-LPF) guidance development to leverage the capabilities properly. The budgeting of this enabling guidance development and subsequent policy implementation is often reported to be lacking in I\&I intensive programs. 


\section{Organizational \& Institutional Obstacles}

Joint teams suffer the additional complications of serving many masters. Each stakeholder commonly will have separate external influences: financial and philosophical, if not statutory, in nature. These issues generate inter- and intra-team dynamics that are unique to I\&I efforts.

3. To what extent do the efforts involve formal plans (e.g., IPT, identified champion) for building and maintaining trust within and among the various teams?

\begin{tabular}{|c|c|c|}
\hline \multirow[t]{4}{*}{ Team interaction } & Team able to produce joint artifacts & $(+)$ \\
\hline & Teams are newly formed & $(-)$ \\
\hline & $\begin{array}{l}\text { Complete \& ongoing participation by } \\
\text { members }\end{array}$ & $(+)$ \\
\hline & Identified champion of joint goals & $(+)$ \\
\hline \multirow[t]{4}{*}{ Veto power } & Independent budget control & $(-)$ \\
\hline & Able to withdraw funding & $(-)$ \\
\hline & 0-3 independent parties & $(+)$ \\
\hline & Greater than 5 independent parties & $(-)$ \\
\hline \multirow[t]{3}{*}{ Conflict reduction } & Risk mitigation plans/strategies exist & $(+)$ \\
\hline & Formal negotiation practices & $(+)$ \\
\hline & No conflict resolution strategies & $(-)$ \\
\hline \multirow[t]{4}{*}{ Arbitration process } & $\begin{array}{l}\text { Defined and agreed to among all the key } \\
\text { teams }\end{array}$ & $(+)$ \\
\hline & Formal process with lead contractor only & $(-)$ \\
\hline & $\begin{array}{l}\text { Each critical team that works on the project } \\
\text { has established and agreed on a formal } \\
\text { process }\end{array}$ & $(+)$ \\
\hline & No formal arbitration process & $(-)$ \\
\hline \multirow[t]{3}{*}{ Formal governance } & Penalties for early termination & $(+)$ \\
\hline & Binding agreements among all stakeholders & $(+)$ \\
\hline & No penalties & $(-)$ \\
\hline \multirow[t]{3}{*}{ Incentives } & Cut across organizational boundaries & $(++)$ \\
\hline & $\begin{array}{l}\text { Money flows from the level that has the } \\
\text { joint I\&I motivation }\end{array}$ & $(+)$ \\
\hline & Based in separate organization's goals & $(-)$ \\
\hline
\end{tabular}




\section{Lifecycle Sustainment}

Lifecycle sustainment in stand-alone software systems is traditionally low risk. However, the interdependencies of highly integrated and interoperable systems do generate sustainment issues-particularly if constituent parts must be independently maintained. Transferring these systems from development to operations is more difficult due to the need to continuously maintain I\&I independencies.

4. Look for evidence that I\&I sustainment activities have been considered.

\begin{tabular}{lll}
\hline Interdependence & Dependencies are documented & $(+)$ \\
& Interactions are sequential in nature & $(++)$ \\
& Interactions are pooled & $(+)$ \\
& Interactions require reciprocal actions & $(-)$ \\
& Well developed relationship models & $(+)$ \\
& Critical functions are loosely ${ }^{18}$ coupled & $(+)$ \\
& Critical functions are tightly coupled & $(-)$ \\
\hline DOTMLPF & Ongoing sustainment budgeted & $(+)$ \\
& Implementation of changes budgeted & $(+)$ \\
& Stakeholder Measured CSU & $(+)$ \\
\hline Semantics & Documented & $(+)$ \\
& Training to institutionalize changes & $(+)$ \\
& Measured Common Shared Understanding & $(+)$ \\
\hline Historically proven & $(+)$ \\
\hline Precedence & Performance measures in place & $(+)$ \\
& Cross DOTMLPF spectrum & $(+)$ \\
\hline No mission critical operational experience & $(--)$ \\
\hline Non-mission critical experience & $(+)$ \\
\hline Demonstrated stability in a mission critical & $(++)$ \\
\hline operational setting & \\
\hline & & $(+)$ \\
\hline
\end{tabular}

5. Unfortunately, the state of practice relies heavily on testing for accomplishing I\&I. This leads to significantly larger testing budget requirements as the test-rework loops perform "brute force" I\&I.

18 Loose coupling often leads to shortcomings in security policies, legacy systems utilization, and complex code requirements. Has the program anticipated these costs?

19 DOTMLPF is doctrine, organization, training, materiel, leadership and education, personnel and facilities. 


\section{Team Performance}

I\&I programs require exceptional team performance in the face of exceptional team composition. Team members often come from disparate organizations with conflicting goals, independent funding, and localized incentives. It takes tremendous leadership and individual commitment and flexibility to achieve synergistic outcomes in such environments.

6. The social constructive nature of establishing I\&I requirements requires highly mature problem solving and coordinating efforts.

\begin{tabular}{lll}
\hline Problem space & Documented, clear understanding & $(+)$ \\
& Measured consensus (CSU) & $(+)$ \\
& Holistic representation & \\
& Demonstrated transference & $(+)$ \\
Experience & Team has proven track record & $(+)$ \\
\hline & Constituents performed in similar SoS & $(+)$ \\
& situations & $(+)$ \\
\hline \multirow{2}{*}{ Formalized coordination } & Clerical assistance & $(+)$ \\
& Dedicated program champion & $(+)$ \\
& Conscientious plan to monitor the & $(+)$ \\
& effectiveness of tools and techniques for & \\
\hline eliminating time and distance problems & \\
\hline Decision behaviours & Snap judgements & $(-)$ \\
& Leaps of faith & $(-)$ \\
& Risk aversion & $(-)$ \\
\hline
\end{tabular}

7. Integration and Interoperability (I\&I) programs may demand synthesis of several domains of knowledge.

\begin{tabular}{lll}
\hline Semantics & Documented & $(+)$ \\
& Measured consensus & $(+)$ \\
\hline Culture & Unvoiced opinion & $(-)$ \\
& Rank disparity & $(-)$ \\
& Multiple Operation Specialties & $(-)$ \\
\hline Disparate demands & Measured Common Shared Understanding & $(+)$ \\
& Intersection $^{21}$ & $(-)$ \\
& Union $^{20}$ & $(+)$ \\
\hline
\end{tabular}

${ }^{20}$ Consensus is responsive to all the needs of the constituents, not a subset.

${ }^{21}$ These are minimal, easier, least-common-denominator solutions. 
42 | CMU/SEI-2010-TR-024 


\section{Appendix B: Additional Analyses Using DAES-SARS Information for Forecasting Performance}

\section{Overview}

This appendix presents additional analyses associated with Study 2 that is described on page 7.

An examination of other statistical techniques did not yield any better insight than the Assessment Indicators that are listed in Table 2 on page 9.

The Earned Value Indicator, for instance, was greatly variable until the end of each phase.

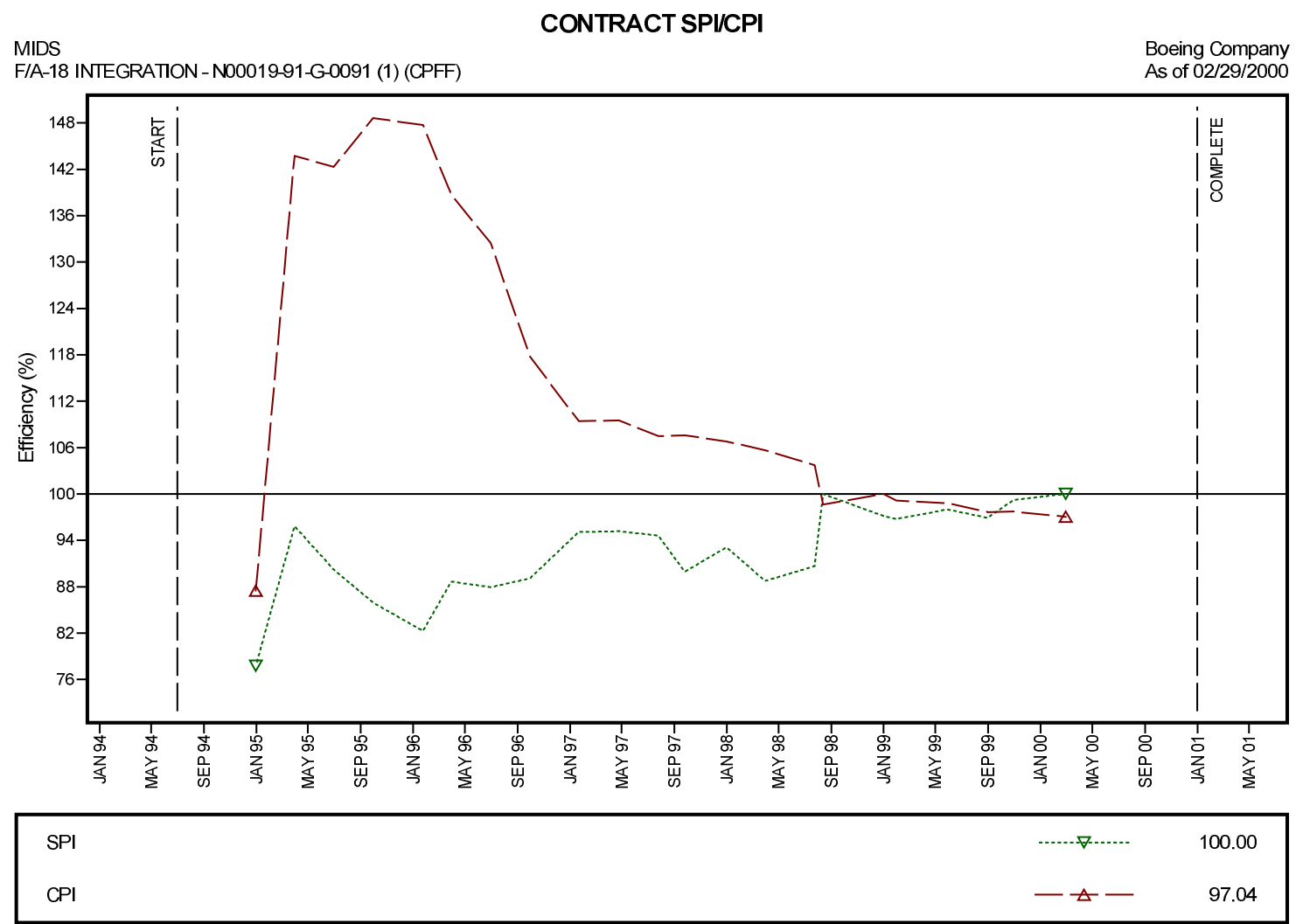

Figure 15: Sample Earned Value Management Report

\section{Text Analysis of Program Review Reports}

The use of data mining tools to extract information was also explored due to the quantity of textual information in the DAES/SARs. The hope was that such tools could reduce the reading load by quickly displaying the important conceptual structure embodied by several years of DAES/SARs. Although we determined that the tools were not adequate for total reliance, the results did confirm the evolution of testing issues into corrective actions observed by our reading and thus served as a confirmation of this complex issue. Otherwise, we found 
little evidence that the executive DAES reports contained significant references to the types of SoS risk factors $^{22}$ identified from the subject matter expert and literature reviews conducted in the early stages of this research. None of these factors are systematically captured by current acquisition reporting requirements. coverage. 


\section{References/Bibliography}

URLs are valid as of the publication date of this document.

\section{[Agranoff 2003]}

Agranoff, Robert and McGuire, Michael. Collaborative Public Management: New Strategies for Local Governments. Georgetown University Press, 2003.

\section{[Brown 2007]}

Brown, M.M., Flowe, R.M., \& Hamel, S.P. "The Acquisition of Joint Programs: The Implications of Interdependencies." CrossTalk May 2007.

http://www.stsc.hill.af.mil/crosstalk/2007/05/0705BrownFloweHamel.html

\section{[CJCS 2000]}

Chairman of the Joint Chiefs of Staff, Strategy Division, Directorate for Strategic Plans and Policy (J-5), Joint Staff. Joint Vision 2020. http://www.dtic.mil/doctrine/jel/jfq_pubs/1225.pdf (2000).

\section{[CJCSI 2005]}

Chairman of the Joint Chiefs of Staff Instruction. Joint Capabilities Integration and Development System. https://acc.dau.mil/GetAttachment.aspx?id=132122\&pname=file\&aid=26664\&lang=en-US (2005).

\section{[CJCSI 2008]}

Chairman of the Joint Chiefs of Staff Instruction. Interoperability and Supportability of Information Technology and National Security Systems. http://www.dtic.mil/cjes_directives/cdata/unlimit/6212_01.pdf (2008).

\section{[CJCS 2009]}

Chairman of the Joint Chiefs of Staff. Capstone Concept for Joint Operations. http://www.jfcom.mil/newslink/storyarchive/2009/CCJO_2009.pdf (2009).

\section{[Cross 2006]}

Cross, Steven M. "Data Analysis and Its Impact on Predicting Schedule and Cost Risk." Thesis, Air Force Institute of Technology, Wright-Patterson Air Force Base, OH, School of Engineering and Management, 2006.

\section{[DoD 2010]}

Department of Defense. Ch. 11.1.2, "Joint Acquisition Management." Defense Acquisition Guidebook. https://acc.dau.mil/CommunityBrowser.aspx?id=325255 2010 (2010).

\section{[DoDAF 2007]}

U.S. Department of Defense. DoD Architecture Framework, Version 1.5, Volume I: Definitions and Guidelines. http://cio-nii.defense.gov/docs/DoDAF_volume_I.pdf (2007).

\section{[DoDD 2004]}

Department of Defense Directive. Data Sharing in a Net-Centric Department of Defense. http://www.dtic.mil/whs/directives/corres/pdf/832002p.pdf (2004). 


\section{[Doyle 2009]}

Doyle, John M. Levin, McCain Introduce Defense Reform Bill. Aviation Week.

http://www.aviationweek.com/aw/generic/story_channel.jsp?channel=defense\&id=news/ACT022509.xml (2009).

\section{[Foreman 2007]}

Foreman, James D. "Predicting the Effect of Longitudinal Variables on Cost and Schedule Performance." Thesis, Air Force Institute of Technology, Wright-Patterson Air Force Base, OH, School of Engineering and Management, 2007.

\section{[Gailey 2002]}

Gailey III, Charles K. Predictive Power for Program Success from Engineering and Manufacturing Development Performance Trends (DAU Press Technical Report, TR 1-02). Fort Belvoir, VA: Defense Acquisition University, Defense Systems Management College, 2002.

http://www.dau.mil/pubs/trpts/TR_1_02.pdf

\section{[GAO 2009]}

United States Government Accountability Office. Defense Acquisition: Charting a Course for Lasting Reform. http://www.gao.gov/new.items/d09663t.pdf (2009).

\section{[Genest 2004]}

Genest, Daniel C. "Logistic and Multiple Regression: The Two-Step Approach to Estimating Cost Growth." Thesis, Air Force Institute of Technology, Wright-Patterson Air Force Base, OH, Department of Mathematics and Statistics, 2004. http://handle.dtic.mil/100.2/ADA423097

\section{[Gordon 1996]}

Gordon, James A. “'Does a Rubber Baseline Guarantee Overruns?' A Study of Cost Performance and Contract Changes in Major Defense Acquisition Programs." Thesis, Air Force Institute of Technology, WrightPatterson Air Force Base, OH, Graduate School of Logistics and Acquisition Management, 1996.

\section{[Grace 1984]}

Grace, J. Peter. War on Waste: President's Private Sector Survey on Cost Control. Macmillan Publishing Co., 1984.

\section{[Kadish 2006]}

Kadish, Ronald (Chairman). Defense Acquisition Performance Assessment Report. https://acc.dau.mil/GetAttachment.aspx?id=17721\&pname=file\&aid=577\&lang=en-US (2006).

\section{[Laszlo-Barabasi 2002]}

Laszlo-Barabasi, Albert. Linked: The New Science of Networks. Perseus Publishing, 2002.

\section{[Lederman 1999]}

Lederman, Gordon Nathaniel. Reorganizing the Joint Chiefs of Staff: The Goldwater-Nichols Act of 1986. Greenwood Press, 1999. 


\section{[Meier 2008]}

Meier Kenneth, Meier J., and O’Toole, Laurence J. "Managerial Networking." Administration \& Society, 37, 5, (2008): 523-541.

\section{[Metcalfe 1995]}

Metcalfe, B. “A Network Becomes More Valuable as it Reaches More Users.” Infoworld (Oct. 1995).

\section{[Morris 1994]}

Morris, E., Levine, L., Meyers, C., Place, P., \& Plakosh, D. System of Systems Interoperability (SOSI) Final Report. (CMU/SEI-2004-TR-004). Pittsburgh, PA: Software Engineering Institute, Carnegie Mellon University, 2004. http://www.sei.cmu.edu/publications/documents/04.reports/04tr004.html

\section{[OIsen 2005]}

Olsen, Johan P. (2005). "Maybe It Is Time to Rediscover Bureaucracy." Journal of Public Administration Research and Theory. http://jpart.oxfordjournals.org/cgi/content/abstract/16/1/1 (2005).

\section{[Packard 1986]}

Packard, Daved. A Quest for Excellence: Final Report to the President.

http://www.ndu.edu/library/pbrc/36ex2.pdf (1986).

\section{[Provan 2007]}

Provan, K.G., Fish, A. and Sydow, J. "Interorganizational Networks at the Network Level: A Review of the Empirical Literature on Whole Networks.”J Journal of Management, 33 (2007):479-516.

\section{[Sipple 2002]}

Sipple, Vincent P. "Estimating Engineering Cost Risk Using Logistic and Multiple Regression.” Thesis, Air Force Institute of Technology, Wright-Patterson Air Force Base, OH, Graduate School of Engineering and Management, 2002. http://handle.dtic.mil/100.2/ADA400576

\section{[Swank 2000]}

Swank, William. J.; Alfieri, Paul A.; Gailey III, Charles K.; \& Reig, Raymond W. Acquisition Trend Metrics in the Department of Defense (DAU Press Technical Report, TR 1-00). Fort Belvoir, VA: Defense Acquisition University, Defense Systems Management College, 2000. http://www.dau.mil/pubs/trpts/tr_1-00.pdf 
48 | CMU/SEI-2010-TR-024 


\title{
REPORT DOCUMENTATION PAGE
}

Form Approved OMB No. 0704-0188

Public reporting burden for this collection of information is estimated to average 1 hour per response, including the time for reviewing instructions, searching existing data sources, gathering and maintaining the data needed, and completing and reviewing the collection of information. Send comments regarding this burden estimate or any other aspect of this collection of information, including suggestions for reducing this burden, to Washington

Headquarters Services, Directorate for information Operations and Reports, 1215 Jefferson Davis Highway, Suite 1204, Arlington, VA 22202-4302, and to the Office of Management and Budget, Paperwork Reduction Project (0704-0188), Washington, DC 20503.

\begin{tabular}{ll|l|}
\hline $\begin{array}{l}\text { 1. } \\
\text { (Leave Blank) }\end{array}$ & $\begin{array}{l}\text { 2. REPORT DATE } \\
\text { July } 2010\end{array}$ \\
\hline 4. & $\begin{array}{l}\text { TITE AND SUBTITE } \\
\text { Programmatic and Constructive Interdependence: Emerging Insights and Predictive Indicators } \\
\text { of Development Resource Demand }\end{array}$
\end{tabular}

\section{REPORT TYPE ANDDATES COVERED}

Final

5. FUNDING NUMBERS

FA8721-05-C-0003

6. AUTHOR(S)

Robert M. Flowe, Mark Kasunic, Mary Maureen Brown, Paul L. Hardin III, James McCurley, David Zubrow, William Anderson

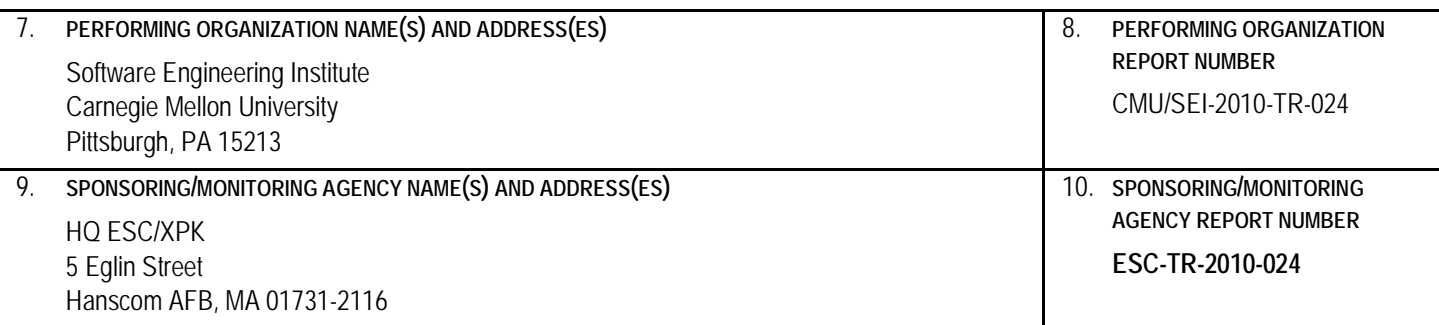

11. SUPPLEMENTARY NOTES

\begin{abstract}
12A DISTRIBUTION/AVAILABIUTY STATEMENT
12B DISTRIBUTIONCODE

Unclassified/Unlimited, DTIC, NTIS

13. ABSTRACT (MAXIMUM 200 WORDS)

The challenges program managers encounter in attempting to deliver programs on time and on budget are well substantiated. $A$ significant driver of the turbulence experienced by acquisition programs today is the transformation to joint capabilities. This report describes a series of ongoing research efforts, sponsored by the Office of the Secretary of Defense (OSD), that investigated the role of interdependence in the acquisition of major defense acquisition programs.

The overall goal of the research was to identify, quantify, and assess the degree of programmatic and constructive interdependence and to assess the effects of interdependence on program risk. A number of important findings and noteworthy insights were discovered as programs were examined in light of their interdependencies with other programs. The results indicate that an expanded definition of interdependencies along with the incorporation of network analysis tools may provide important insights into program performance in a joint capability arena.
\end{abstract}

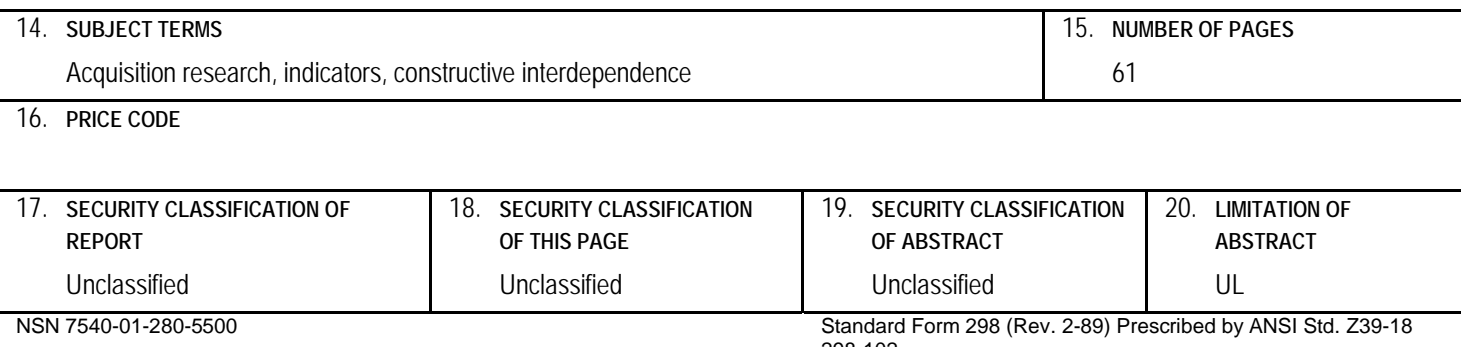

NSN 7540-01-280-5500 